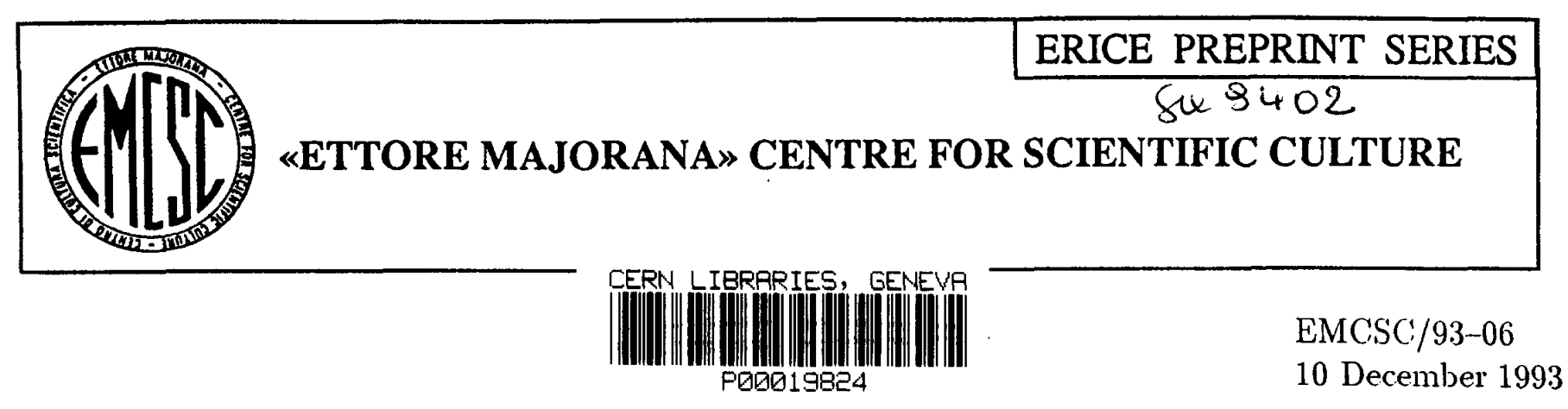

\title{
MONTE CARLO SIMULATIONS FOR LEADING PROTON PRODUCTION IN DEEP INELASTIC SCATTERING
}
A. V. Anisovich ${ }^{3,9,11}$, G. Anzivino 4 , F. Arzarello ${ }^{2}$, G. Bari ${ }^{2}$, M. Basile ${ }^{2,7}$,
L. Bellagamba ${ }^{2}$, D. Boscherini' ${ }^{2}$, G. Bruni ${ }^{2}$, P. Bruni ${ }^{2}$, G. Cara Romeo ${ }^{2}$, M. Chiarini ${ }^{2,11}$, L. Cifarelli ${ }^{2,8}$, F. Cindolo ${ }^{2}$, F. Ciralli ${ }^{2}$, A. Contin ${ }^{1,2}$, M. Dardo ${ }^{5,10}$, S. D'Auria ${ }^{2}$, C. Del Papa ${ }^{2,7}$, S. De Pasquale ${ }^{4}$, F. Frasconi ${ }^{2}$, P. Giusti ${ }^{2}$, G. Iacobucci ${ }^{2}$, G. Maccarrone ${ }^{4}$, A. Margotti ${ }^{2}$, T. Massam ${ }^{2}$, R. Nania ${ }^{2}$, S. Qian ${ }^{2,11}$, G. Sartorelli2,7, Yu. M. Shabelski ${ }^{3,9,11}$, S. Yu. Sivoklokov ${ }^{3,6,11}$, M. A. Stepanov ${ }^{3,9,11}$, R. Timellini ${ }^{2,11}$, L. Votano ${ }^{4}$ and A. Zichichi ${ }^{1,2}$

1) CERN, Geneva, Switzerland

2) INFN, Bologna, Italy

3) INFN, Eloisatron Project, Erice; Italy

4) INFN, LNF, Frascati, Italy

5) INFN, Torino, Italy

6) INP, Moscow State University, Moscow, Russia

7) Physics Department, University of Bologna, Italy

8) Physics Department, University of Pisa, Italy

9) PNPI, Gatchina, St. Petersburg, Russia

10) II Faculty of Science, University of Torino, Alessandria, Italy

11) World Laboratory, Lausanne, Switzerland

\begin{abstract}
Predictions for "leading" proton production in Deep Inelastic Scattering at HERA energies are presented in the framework of different Monte Carlo event generators, i.e. LEPTO, HERWIG and PYTHIA, in terms of Feynman- $x$ and transverse momentum distributions. In order to get a comprehensive picture of the various possibilities, different structure functions, different $Q^{2}$-values, different reference frames and different types of events (minimum bias events and events with charm and beauty, separately) are considered.
\end{abstract}

(Submitted to Il Nuovo Cimento)

EMCSC - Via Guarnotta 26 - 91016 Erice - Italy - Tel. (923) 869133 - Fax. (923) 869226 

The Leading Proton Spectrometer (LPS) of the ZEUS detector [1] at HERA is an essential tool to continue, in $e p$ Deep Inelastic Scattering (DIS) at high energies, the study of the "leading effect", discovered and first studied at ISR [2-27].

In the present paper we consider the predicted $x_{F}$ (Feynman- $x$ ) and $p_{T}^{2}$-distributions of secondary protons as obtained with three different Monte Carlo event generators: LEPTO (version 6.1) [28], HERWIG (version 5.5) [29] and PYTHIA (version 5.6) [30]. There is a significant difference between these event generators, in particular as far as hadronization is concerned. LEPTO and PYTHIA are both based on the ideas of the Lund String Model, but they are differently structured at the stage of hard processes. LEPTO allows one to consider the DIS process as a function of $Q^{2}$ and separately in the $e p$ frame (the real laboratory frame) and in the $\gamma^{*} p$ frame (a more physical one), where $\gamma^{*}$ is the exchanged neutral vector-particle. In PYTHIA the so-called $Q^{2}$-parameter is not directly related to the four-momentum transfer of DIS in terms of kinematical variables. It represents the effective hardness of the interaction and provides the structure function scale. HERWIG contains the real $Q^{2}$-dependence of DIS processes (as LEPTO), but it is based on different physical ideas, being the Monte Carlo version of a particular Cluster Model.

A detailed comparison of the results from these three generators is presented herein. The agreement among the various predictions can illustrate today's generally accepted view on high-energy neutral-current DIS phenomena. Any disagreement can reflect the real difference in the physical pictures described by the three models and therefore suggest interesting experimental tests.

\section{FEYNMAN- $x$ DISTRIBUTION OF SECONDARY PROTONS IN DIS}

Let us first consider the Feynman- $x\left(x_{F}\right)$ distribution of secondary protons produced in $e p$ collisions at HERA energy $(\sqrt{s}=314 \mathrm{GeV})$. We use the invariant inclusive crosssection ${ }^{1)} x_{E} / \sigma_{\epsilon v} \cdot d \sigma / d x_{F}$, where $x_{E}=2 E / \sqrt{s}, x_{F}=2 p_{L} / \sqrt{s}\left(E\right.$ and $p_{L}$ being the proton energy and longitudinal momentum, respectively) and $\sigma_{e v}$ is the total inelastic crosssection, for each class of events considered. In fig. 1a we present the predictions of LEPTO, HERWIG and PYTHIA in the ep centre-of-mass frame for all DIS events. Here and in the following, unless differently specified, LEPTO and HERWIG events are generated at $Q^{2} \geq$ $4 \mathrm{GeV}^{2}$. Notice that $4 \mathrm{GeV}^{2}$ is the minimum $Q^{2}$-value allowed in LEPTO and also the minimum scale value usable for most of structure function parametrizations. In PYTHIA we use the default $Q^{2}$-parameter setting: this corresponds to an effective $Q^{2}$-scale $\geq 4$ $\mathrm{GeV}^{2}$ and to a distribution of DIS $Q^{2}$-values with $<Q^{2}>\sim 2 \mathrm{GeV}^{2}$, where roughly $1 / 2$ of the events have $Q^{2}<1 \mathrm{GeV}^{2}$. A well-defined "leading effect" appears in fig. 1a, since most of the protons are produced at positive $x_{F}$-values (i.e. in the direction of the incident proton). The LEPTO and PYTHIA distributions are overlapped and show a plateau in the $x_{F}>0$ region, centred around $x_{F} \sim 0.5$ (as in the case of proton-proton collisions, in each

1) For this cross-section, Feynman scaling should be approximately valid. 
$x_{F}$-hemisphere [31]). It should be pointed out that LEPTO predictions fit reasonably well the $x_{F}$-distributions of protons and antiprotons measured in $\mu p$ DIS at lower energy [32]. The HFRWIG distribution has a narrower plateau, centred around $x_{F} \sim 0.35$ and rapidly decreasing at relatively high $x_{F}$, thus showing a less pronounced "leading effect". In fig. 1a the EHLQ1 [33] set of parton structure functions was used. To check the influence of structure functions, in fig. 1b we present the same distributions obtained with set MT (S-DIS) [34]: although the shapes of the distributions slightly change, the difference between fig. $1 \mathrm{a}$ and fig. $1 \mathrm{~b}$ is not significant and in the following we will always use set EHLQ1. This set is rather "old", however it seems to be more adequate in the case of PYTHIA calculations when these are compared with low-energy data on charm and beauty production in $p p$ collisions [35]. In fig. 1c we show the same spectrum as in fig. 1a once the contribution of "non-leading" protons, i.e. those protons which are pair-produced in the central region together with antiprotons, has been subtracted. To do so we actually subtract from the inclusive $x_{F}$-spectrum relative to all protons the corresponding spectrum relative to all antiprotons. The proton spectrum of fig. 1a is practically unaffected by this subtraction in the positive $x_{F}$-hemisphere, say at $x_{F}>0.1$ (fig. 1c), and keeps its relevant figures, in particular in the forward region at $x_{F}>0.5$. In the following we will always consider inclusive distributions, relative to all secondary protons. The same distributions as in fig. 1a are presented in terms of $1 / \sigma_{e v} \cdot d \sigma / d x_{F}$ in fig. $1 \mathrm{~d}$ where the "non-leading" proton contribution clearly shows up.

Let us now consider the $Q^{2}$-dependence of the proton $x_{F}$-distribution. As already mentioned, the calculations at different $Q^{2}$-values, with $Q^{2}$ directly connected with the incident and scattered electron kinematical variables, can only be performed with LEPTO and HERWIG. These are shown in figs. $2 \mathrm{a}$ and $2 \mathrm{~b}$, respectively, at three different $Q^{2}$ values: 7,100 and $1000 \mathrm{GeV}^{2}$. In both cases, the distributions are shifted towards smaller $x_{F}$ as $Q^{2}$ increases: in fact the central production (at $x_{F}<0.1$ ) increases, while the very forward production in the positive $x_{F}$-region decreases, i.e. the "leading effect" decreases. In LEPTO this shift is more significant than in HERWIG. The same $x_{F}$-distributions obtained with PYTHIA at different $Q^{2}$ are presented in fig. 2c. Here the nominal values of $Q^{2}\left(7,100\right.$ and $\left.1000 \mathrm{GeV}^{2}\right)$ actually correspond to the selection of the previously mentioned $Q^{2}$-parameter in the following intervals: $4 \div 10,80 \div 120$ and $800 \div 1200 \mathrm{GeV}^{2}$. There is no significant difference between PYTHIA and LEPTO predictions.

The reason for the shift is rather simple. The main contribution to the DIS crosssection at small $x_{B}$ (Bjorken- $x$ ) comes from the $\gamma^{*}$ interaction with a sea quark (or antiquark) produced via gluon cascade. When $x_{B}$ increases, the $\gamma^{*}$ interacts with a valence quark of the initial proton with higher probability, so the average number of spectator valence quarks which can recombine into a "leading" proton decreases.

It is also interesting to study the behaviour of secondary protons in DIS events when heavy flavours ( $c \bar{c}$ and $b \bar{b}$ ) are produced. The results with LEPTO, HERWIG and PYTHIA are presented in figs. $3 \mathrm{a}$ and $3 \mathrm{~b}$, for charm and beauty, respectively. When comparing them with those in fig. 1a, it appears that in both HERWIG and PYTHIA the "leading" proton production at high positive $x_{F}$ is only slightly affected by the presence of heavy flavours, while it is clearly reduced in LEPTO. The overall effect of heavy flavour production can be better visualized when considering the average $x_{F}$ of secondary protons, as reported in table 1 for the three event generators considered herein. According to LEPTO, the 
proton $\left\langle x_{F}\right\rangle$ decreases by $35 \%$ in $c \bar{c}$ events with respect to all (i.e. minimum bias) DIS events. This shift is only $11 \%$ in PYTHIA and $6 \%$ in HERWIG. When going from $c \bar{c}$ to $b \bar{b}$ events, $\left\langle x_{F}\right\rangle$ slightly decreases (by only a few $\%$, within the statistical errors) in any of the three event generators, thus showing a weak dependence on the heavy quark mass. Notice that, contrary to fig. 1a, in figs. $3 \mathrm{a}$ and $3 \mathrm{~b}$ the LEPTO and HERWIG predictions are closer and significantly differ from the PYTHIA ones.

In $e p$ interactions, if the main contribution to heavy flavour production is given by the photon-gluon fusion subprocess $\gamma^{*} g \rightarrow Q \bar{Q}, Q \bar{Q}(c \bar{c}$ or $b \bar{b})$ pairs are predominantly produced in the central (or photon fragmentation) region ${ }^{2)}$. And these are mostly meson pairs which hardly decay into protons (and antiprotons). A reduced "leading" proton production in the presence of heavy flavours, as observed in particular with LEPTO, indicates a change in the recombination mechanism of spectator valence quarks with respect to all DIS events, which is likely related to the higher values of $Q^{2}$ involved when the $\gamma^{*}$ interacts with a heavier sea quark or antiquark. In fact with LEPTO one obtains $\left\langle Q^{2}\right\rangle=27,40$ and $77 \mathrm{GeV}^{2}$ in all, $c \bar{c}$ and $b \vec{b}$ events containing at least one proton, respectively. The influence of heavy flavour production on the proton $x_{F}$-distribution also appears when simulating $p p$ interactions. Moreover, the energy level and the choice of structure functions seem to play a role in this respect, as discussed elsewhere [36].

If $\left\langle Q^{2}\right\rangle$ increases when going from minimum bias to heavy-flavoured events, it is also instructive to check whether the $Q^{2}$-dependence of the proton $x_{F}$-distribution is different for these two classes of events. In fig. 4 we show for instance the proton $x_{F^{-}}$ distribution in events with charm, obtained with LEPTO at $Q^{2}=7,100$ and $1000 \mathrm{GeV}^{2}$ as in fig. 2a for all DIS events. Contrary to fig. 2a, in fig. 4 there is no clear effect of $Q^{2}$ on the production of "leading" protons at high $x_{F}$.

This fact gives an additional argument to our previous explanation of the shift observed in fig. 2a: for charm production, when the $\gamma^{*} g$ fusion subprocess is at work, with the $\gamma^{*}$ always interacting with a $c$ (or $\bar{c}$ ) sea quark, the average number of spectator valence quarks cannot depend significantly on $Q^{2}$. The same holds true for $b($ or $\bar{b})$ quarks.

Finally, the proton spectra of fig. 2a, obtained with LEPTO for all DIS events at different $Q^{2}$-values in the $e p$ centre-of-mass frame, are again presented in fig. 5a but in the $\gamma^{*} p$ frame. At $x_{F}>0$ the curves of fig. 5a compare well with the corresponding ones in fig. 2a. Namely the "leading effect" is still evident and the $Q^{2}$-dependence is similar. At $x_{F}<0$, the spectra of fig. 5a show a small "shoulder" (around $x_{F} \sim-0.1$ ) which slightly grows as $Q^{2}$ increases. This shoulder contains the already mentioned "nonleading" protons from centrally produced $p \bar{p}$ pairs. In fact the corresponding antiproton spectra, shown in fig. $5 \mathrm{~b}$, are peaked exactly in the same $x_{F}$-region.

To summarize our results, in table 2 we give the average $x_{F}$-values vs. $Q^{2}$ relative to all secondary protons produced in the various conditions discussed so far.

2) We will further discuss this point in section 3 (see fig. 7). 


\section{TRANSVERSE MOMENTUM DISTRIBUTION OF SECONDARY PROTONS}

Let us consider the transverse momentum distribution of protons produced in DIS final states, in terms of $1 / \sigma_{e v} \cdot d \sigma / d p_{T}^{2}$. LEPTO, PYTHIA, and HERWIG predictions are shown in figs. $6 \mathrm{a}, 6 \mathrm{~b}$ and $6 \mathrm{c}$, respectively, for three $x_{F}$-intervals: $0<x_{F}<0.5$, $0.5<x_{F}<1$ and $0<x_{F}<1$ (i.e. the whole positive $x_{F}$-range). The results refer to the $\epsilon p$ frame, with EHLQ1 structure functions. The predictions from the three event generators are quite different. LEPTO (fig. 6a) predicts higher $p_{T}$ for protons with $x_{F}<0.5$ than for protons with $x_{F}>0.5$ : the difference in slope of the corresponding distributions is significantly large. In PYTHIA (fig. 6b) this difference still exists, but smaller, while in HERWIG (fig. $6 \mathrm{c}$ ) the $p_{T}^{2}$-distributions at low and high $x_{F}$ are really the same. In fig. $6 \mathrm{~d}$ the PYTHIA, LEPTO and HERWIG $p_{T}^{2}$-distributions relative to "leading" protons with $x_{F}>0.5$ are superimposed for comparison: the HERWIG curve is much less steep than the other two which are degenerate. In the whole $x_{F}>0$ range, the slope of the $p_{T}^{2}$-spectrum decreases when going from PYTHIA (fig. 6b) to LEPTO (fig. 6a) and to HERWIG (fig. $6 \mathrm{c})$. Notice that the spectra corresponding to $0<x_{F}<1$ and $0<x_{F}<0.5$ are obviously almost the same since the bulk of secondary protons are produced at low $x_{F}$. The average $p_{T}^{2}$-values of the distributions presented in fig. 6 for $x_{F}>0$ and $x_{F}>0.5$, are reported in table 3 .

The nature of the difference in $p_{T}$ for protons having large and small $x_{F}$, as predicted by LEPTO and PYTHIA generators, seems to be clear enough. We know that at small $x_{B}$ the $\gamma^{*}$ mainly interacts with a sea quark (or antiquark) produced via gluon cascade. An example of QCD diagram is presented in fig. 7 . Here we can see the gamma-gluon interaction in the top part of the diagram with the production of a sea $q_{s} \bar{q}_{s}$ pair. The upper gluon emits other gluons and looses its virtuality and transverse momentum. The lower gluon is absorbed by a valence quark $q_{v}$ of the proton. The emitted gluons also are converted into $q_{s} \bar{q}_{s}$ pairs. On the last step, all produced quarks are converted into hadrons and the probability for two or three quarks to recombine into one hadron decreases exponentially with increasing rapidity gap between these quarks. So valence quarks most probably recombine with sea quarks from the lower part of the diagram in fig. 7 , which have comparatively small transverse momenta. As a result, the average transverse momentum of "leading" secondary hadrons (protons with high $x_{F}$ ) should be comparatively small too. On the other hand, the production of secondary protons with small $x_{F}$ is due to the fusion of sea quarks from the upper and middle parts of the diagram, so these protons should have higher transverse momenta.

Let us now study how the proton $p_{T}$ varies as a function of $Q^{2}$, using first either LEPTO or HERWIG. Since the shapes of the $p_{T}^{2}$-spectra obtained with these two event generators turn out to be more or less $Q^{2}$-independent, we will focus the discussion on the average $p_{T}^{2}$. Figures $8 \mathrm{a}$ and $8 \mathrm{~b}$, corresponding to LEPTO and HERWIG, respectively, illustrate the variation of $\left\langle p_{T}^{2}>\right.$ vs. $Q^{2}$ for protons with $0<x_{F}<0.5,0.5<x_{F}<1$ and $0<x_{F}<1$. Three $Q^{2}$-values are considered $\left(7,100\right.$ and $\left.1000 \mathrm{GeV}^{2}\right)$, as in section 2. Unless differently specified, the data refer to all DIS events and to the ep centre-ofmass frame. LEPTO and HERWIG results are quite similar: for protons produced at low $x_{F}$ (and therefore for all protons), $<p_{T}^{2}>$ increases very rapidly with $Q^{2}$; for "leading" 
protons with higher $x_{F},\left\langle p_{T}^{2}\right\rangle$ does not practically depend on $Q^{2}$. Analogous results obtained with PYTHIA are shown in fig. 8c. They differ from LEPTO predictions, which can be partly related with the particular definition of $Q^{2}$ in PYTHIA.

The growth of $\left\langle p_{T}^{2}\right\rangle$ for protons with $x_{F}<0.5$ is mainly connected with pure kinematical reasons. When $Q^{2}$ increases, the transverse momentum of $\gamma^{*}$ also increases. As a consequence, the $\gamma^{*} p$ frame (which is the physical one) acquires some transverse boost relative to the $e p$ frame. This translates into an increase of transverse momentum in the $e p$ frame for all secondaries produced at small $x_{F}$. For secondaries with large $x_{F}$, this effect is practically absent. To illustrate this point, in figs. $9 \mathrm{a}$ and $9 \mathrm{~b}$ we present the predictions of LEPTO for $\left\langle p_{T}^{2}>\right.$ vs. $Q^{2}$ in both $e p$ and $\gamma^{*} p$ frames, for protons produced in the central and forward regions, separately. In the $\gamma^{*} p$ frame, the growth of $\left\langle p_{T}^{2}\right\rangle$ with $Q^{2}$ for secondary protons with $x_{F}<0.5$ (fig. $9 \mathrm{a}$ ) is much weaker than in the ep frame and it is absent, as in the $e p$ frame, for protons with $x_{F}>0.5$ (fig. $9 \mathrm{~b}$ ). The results from LEPTO in the $\gamma^{*} p$ frame are summarized in fig. 10.

Let us turn now to heavy flavour production. Qualitatively the same $<p_{T}^{2}>$ vs. $Q^{2}$ trend applies to DIS events containing heavy flavours. Namely the values of $\left\langle p_{T}^{2}\right\rangle$ do not depend on $Q^{2}$ for secondary protons with $x_{F}>0.5$, as shown in figs. $11 \mathrm{a}$ and $11 \mathrm{~b}$ for charm and beauty events generated with LEPTO: On the other hand, $\left\langle p_{T}^{2}\right\rangle$ increases with $Q^{2}$ for protons at small $x_{F}$ but not as strongly as in fig. 8a, relative to all DIS events.

In fact, assuming again that the main subprocess for heavy quark pair-production is $\gamma^{*} g \rightarrow Q \bar{Q}$, then the $Q \bar{Q}$ pair is produced in the upper part of the diagram shown in fig. 7 . Since $c$ or $b$-mesons are much more abundantly produced than $c$ or $b$-baryons, fragmentation and recombination effects are such that the bulk of secondary protons (not coming from heavy baryon decays) are produced on the average closer to the "target" proton. Therefore physical as well as kinematical $Q^{2}$-effects for secondary protons in events with heavy flavours should be smaller.

Again, to summarize our results, the average $p_{T}^{2}$-values vs. $Q^{2}$ discussed in this section are reported all together in tables 3 and 4 .

\section{CONCLUSION}

We have considered three Monte Carlo event generators based on QCD (PYTHIA, LEPTO and HERWIG) to simulate the production of secondary protons in $e p$ Deep Inelastic Scattering at HERA energies.

All of them manifest the "leading proton effect", i.e. an abundant proton production at $x_{F}>0$ in the $e p$ centre-of-mass reference frame, with a sizable fraction of protons in the forward region at $x_{F}>0.5$. When all DIS events (i.e. minimum bias events) are considered, this "leading effect" is more pronounced in LEPTO and PYTHIA than in HERWIG. In LEPTO and PYTHIA it decreases as $Q^{2}$ increases, more rapidly than in HERWIG. When only events containing heavy flavours (charm and beauty) are considered, the "leading effect" is also reduced, more in LEPTO than in either PYTHIA or HERWIG, almost independently from the heavy quark mass. In addition, as observed with LEPTO, there 
is no clear $Q^{2}$-clependence of the effect in this case.

As far as the proton transverse momentum is concerned, HERWIG predicts higher $p_{T}$ than LEPTO and PYTHIA for all protons with positive $x_{F}$. For "leading" protons with $x_{F}>0.5$, HERWIG predicts the same $p_{T}$ as for all protons, while LEPTO and PYTHIA predict lower $p_{T}$ (as one would expect, see section 3). In both LEPTO and HERWIG the $p_{T}$ considerably increases with $Q^{2}$ for all protons, but for protons with $x_{F}>0.5$ it turns out to be $Q^{2}$-independent in LEPTO and slightly decreasing with increasing $Q^{2}$ in HERWIG. The same features remain when heavy flavours are produced, although the above $p_{T}$-increase with $Q^{2}$ is much smaller. Finally, compared to the minimum bias case, in LEPTO the proton $p_{T}$ increases with the mass of the produced heavy quark for all protons and in particular for "leading" protons at high $x_{F}$ (see table 3). In PYTHIA and HERWIG there is no clear dependence on the mass.

All the differences observed herein among the results from the various QCD event generators are likely related to differences existing at the hadronization level. A detailed comparison with experimental data cannot but improve the existing Monte Carlo models.

\section{ACKNOWLEDGEMENTS}

We are grateful to Ya. I. Azimov, V. A. Khoze and M. G. Ryskin for interesting discussions. 
The average $x_{F}$-values of secondary protons produced in $e p$ interactions at $\sqrt{s}=314 \mathrm{GeV}$ calculated with LEPTO, PYTHIA and HERWIG using EHLQ1 [33] or MT(S-DIS) [34] structure functions, for all DIS events (i.e. minimum bias) and for events with charm and beauty. The data refer to the $e p$ centre-of-mass frame.

\begin{tabular}{|c||c|c|c|}
\hline & $\left\langle x_{F}\right\rangle$ LEPTO & $\left\langle x_{F}\right\rangle$ PYTHIA & $\left\langle x_{F}\right\rangle$ HERWIG \\
\hline \hline EHLQ1, all & $0.276 \pm 0.003$ & $0.267 \pm 0.004$ & $0.203 \pm 0.002$ \\
\hline MT(S-DIS), all & $0.259 \pm 0.003$ & $0.265 \pm 0.003$ & $0.202 \pm 0.006$ \\
\hline EHLQ1, with $c \bar{c}$ & $0.179 \pm 0.002$ & $0.237 \pm 0.002$ & $0.190 \pm 0.002$ \\
\hline EHLQ1, with $b \bar{b}$ & $0.172 \pm 0.008$ & $0.235 \pm 0.002$ & $0.182 \pm 0.002$ \\
\hline
\end{tabular}

Table 2

The average $x_{F}$-values of secondary protons produced in $e p$ interactions at $\sqrt{s}=314 \mathrm{GeV}$ calculated with LEPTO and HERWIG using EHLQ1 [33] structure functions, for different values of $Q^{2}$. The data refer to all DIS events (i.e minimum bias) and to events with charm and beauty production. Both the $e p$ and $\gamma^{*} p$ centre-of-mass frames are considered, as specified.

\begin{tabular}{|c||c|c|}
\hline$Q^{2}\left[\mathrm{GeV}^{2}\right]$ & $\left\langle x_{F}\right\rangle$ LEPTO & $\left\langle x_{F}\right\rangle$ HERWIG \\
\hline \hline All, $e p$ frame & & \\
7 & $0.279 \pm 0.002$ & $0.204 \pm 0.002$ \\
100 & $0.247 \pm 0.002$ & $0.196 \pm 0.002$ \\
1000 & $0.223 \pm 0.002$ & $0.183 \pm 0.002$ \\
\hline With $c \bar{c}, e p$ frame & & \\
$\tau$ & $0.179 \pm 0.001$ & - \\
100 & $0.183 \pm 0.001$ & - \\
1000 & $0.185 \pm 0.001$ & - \\
With $b \bar{b}, e p$ frame & & - \\
7 & $0.16 \pm 0.01$ & - \\
100 & $0.184 \pm 0.007$ & - \\
1000 & $0.177 \pm 0.005$ & - \\
\hline All,$\gamma^{*} p$ frame & & - \\
100 & $0.316 \pm 0.002$ & - \\
1000 & $0.276 \pm 0.002$ & - \\
\hline
\end{tabular}


Table 3

The average $p_{T}^{2}$-values of secondary protons produced in $e p$ interactions at $\sqrt{s}=314 \mathrm{GeV}$ calculated with LEPTO, PYTHIA and HERWIG using EHLQ1 [33] structure functions, for all DIS events (i.e. minimum bias) and for events with charm and beauty. The data refer to the $e p$ centre-of-mass frame.

\begin{tabular}{|c||c|c|c|}
\hline & $\begin{array}{c}\text { LEPTO } \\
<p_{T}^{2}>\left[\mathrm{GeV}^{2}\right]\end{array}$ & $\begin{array}{c}\text { PYTHIA } \\
<p_{T}^{2}>\left[\mathrm{GeV}^{2}\right]\end{array}$ & $\begin{array}{c}\text { HERWIG } \\
<p_{T}^{2}>\left[\mathrm{GeV}^{2}\right]\end{array}$ \\
\hline \hline All & & & \\
$0<x_{F}<1$ & $0.65 \pm 0.03$ & $0.38 \pm 0.02$ & $0.75 \pm 0.01$ \\
$0.5<x_{F}<1$ & $0.27 \pm 0.01$ & $0.27 \pm 0.01$ & $0.76 \pm 0.02$ \\
\hline With $c \bar{c}$ & & & \\
$0<x_{F}<1$ & $0.57 \pm 0.02$ & $0.33 \pm 0.01$ & $0.63 \pm 0.01$ \\
$0.5<x_{F}<1$ & $0.37 \pm 0.02$ & $0.24 \pm 0.01$ & $0.63 \pm 0.05$ \\
\hline With $b \bar{b}$ & & & \\
$0<x_{F}<1$ & $0.85 \pm 0.12$ & $0.58 \pm 0.03$ & $0.72 \pm 0.02$ \\
$0.5<x_{F}<1$ & $1.0 \pm 0.3$ & $0.26 \pm 0.04$ & $0.54 \pm 0.04$ \\
\hline
\end{tabular}


The average $p_{T}^{2}$-values of secondary protons produced in $e p$ interactions at $\sqrt{s}=314$ GeV calculated with LEPTO and HERWIG using EHQL1 [33] structure functions, for different values of $Q^{2}$. The data refer to all DIS events (i.e minimum bias) and to events with charm and beauty, in the ep centre-of-mass frame.

\begin{tabular}{|c|c|c|c|c|}
\hline & $Q^{2}\left[\mathrm{GeV}^{2}\right]$ & $x_{F}$ & $\begin{array}{c}\text { LEPTO } \\
<p_{T}^{2}>\left[\mathrm{GeV}^{2}\right]\end{array}$ & $\begin{array}{c}\text { HERWIG } \\
<p_{T}^{2}>\left[\mathrm{GeV}^{2}\right]\end{array}$ \\
\hline \multirow{6}{*}{ 三 } & 7 & $0 \div 1$ & $0.43 \pm 0.01$ & $0.63 \pm 0.01$ \\
\hline & 7 & $0.5 \div 1$ & $0.28 \pm 0.04$ & $0.85 \pm 0.02$ \\
\hline & 100 & $0 \div 1$ & $1.33 \pm 0.04$ & $1.33 \pm 0.02$ \\
\hline & 100 & $0.5 \div 1$ & $0.30 \pm 0.03$ & $0.69 \pm 0.02$ \\
\hline & 1000 & $0 \div 1$ & $3.7 \pm 0.1$ & $3.47 \pm 0.01$ \\
\hline & 1000 & $0.5 \div 1$ & $0.27 \pm 0.01$ & $0.64 \pm 0.02$ \\
\hline \multirow{6}{*}{$\begin{array}{l}\text { 导 } \\
\text { 表 } \\
3\end{array}$} & 7 & $0 \div 1$ & $0.42 \pm 0.01$ & - \\
\hline & 7 & $0.5 \div 1$ & $0.32 \pm 0.01$ & - \\
\hline & 100 & $0 \div 1$ & $0.80 \pm 0.02$ & - \\
\hline & 100 & $0.5 \div 1$ & $0.31 \pm 0.01$ & - \\
\hline & 1000 & $0 \div 1$ & $2.04 \pm 0.07$ & - \\
\hline & 1000 & $0.5 \div 1$ & $0.35 \pm 0.02$ & - \\
\hline \multirow{6}{*}{$\begin{array}{l}10 \\
\frac{7}{3} \\
3 \\
3\end{array}$} & 7 & $0 \div 1$ & $0.63 \pm 0.01$ & - \\
\hline & 7 & $0.5 \div 1$ & $0.38 \pm 0.04$ & - \\
\hline & 100 & $0 \div 1$ & $0.82 \pm 0.06$ & - \\
\hline & 100 & $0.5 \div 1$ & $0.39 \pm 0.08$ & - \\
\hline & 1000 & $0 \div 1$ & $1.86 \pm 0.1$ & - \\
\hline & 1000 & $0.5 \div 1$ & $0.29 \pm 0.02$ & - \\
\hline
\end{tabular}




\section{Figure captions}

Fig. $1: x_{F}$-distributions for secondary protons produced in $e p$ interactions at $\sqrt{s}=314$ $\mathrm{GeV}$, as obtained with LEPTO, PYTHIA and HERWIG: all protons with EHLQ1 structure functions (a); all protons with MT (S-DIS) structure functions (b); "leading" protons with EHLQ1 structure functions (c); same as (a) without $x_{E^{-}}$ scaling $(d)$.

Fig. $2: x_{F}$-distributions in the $e p$ centre-of-mass frame for all secondary protons produced in $e p$ interactions at $\sqrt{s}=314 \mathrm{GeV}$ and different $Q^{2}$-values, as obtained with LEPTO (a), HERWIG (b) and PYTHIA (c) using EHLQ1 structure functions.

Fig. $3: x_{F}$-distributions in the $e p$ centre-of-mass frame for all secondary protons produced in $e p$ interactions at $\sqrt{s}=314 \mathrm{GeV}$ in events with charm (a) and beauty (b), as obtained with LEPTO, PYTHIA and HERWIG using EHLQ1 structure functions.

Fig. $4: x_{F}$-distributions in the $\epsilon p$ centre-of-mass. frame for all secondary protons produced in $e p$ interactions at $\sqrt{s}=314 \mathrm{GeV}$ and different $Q^{2}$-values in events with charm, as obtained with LEPTO using EHLQ1 structure functions.

Fig. 5: $x_{F}$-distributions in the $\gamma^{*} p$ centre-of-mass frame for all secondary protons (a) and antiprotons (b) produced in $e p$ interactions at $\sqrt{s}=314 \mathrm{GeV}$ and different $Q^{2}$-values, as obtained with LEPTO using EHLQ1 structure functions.

Fig. $6: p_{T}^{2}$-distributions in the $e p$ centre-of-mass frame for all secondary protons produced in $e p$ interactions at $\sqrt{s}=314 \mathrm{GeV}$ in different $x_{F}$-regions, as obtained with LEPTO (a), PYTHIA (b) and HERWIG (c) using EHLQ1 structure functions; in the forward region $\left(0.5<x_{F}<1\right)$, the LEPTO, PYTHIA and HERWIG distributions are also shown together for comparison $(\mathrm{d})$.

Fig. 7 : An example of QCD diagram for $e p$ DIS at small $x_{B}$.

Fig. $8:<p_{T}^{2}>$ vs. $Q^{2}$ in the $e p$ centre-of-mass frame for all secondary protons produced in $e p$ interactions at $\sqrt{s}=314 \mathrm{GeV}$ in different $x_{F}$-regions, as obtained with LEPTO (a), HERWIG (b) and PYTHIA (c) using EHLQ1 structure functions.

Fig. $9:<p_{T}^{2}>$ vs. $Q^{2}$ in the $e p$ and $\gamma^{*} p$ centre-of-mass frames for all secondary protons produced in $e p$ interactions at $\sqrt{s}=314 \mathrm{GeV}$ with $0<x_{F}<0.5$ (a) and $0.5<x_{F}<1$ (b), as obtained with LEPTO using EHLQ1 structure functions.

Fig. $10:\left\langle p_{T}^{2}>\right.$ vs. $Q^{2}$ in the $\gamma^{*} p$ centre-of-mass frame for all secondary protons produced in $e p$ interactions at $\sqrt{s}=314 \mathrm{GeV}$ in different $x_{F}$-regions, as obtained with LEPTO using EHLQ1 structure functions.

Fig. $11:\left\langle p_{T}^{2}\right\rangle$ vs. $Q^{2}$ in the $e p$ centre-of-mass frame for all secondary protons produced in $\epsilon p$ interactions at $\sqrt{s}=314 \mathrm{GeV}$ in different $x_{F}$-regions when charm (a) and 
beauty (b) are also produced, as obtained with LEPTO using EHLQ1 structure functions. 


\section{REFERENCES}

[1] ZEUS Collaboration (M. Derrick et al.): "The ZEUS Detector: Status Report 1989", DESY PRC 89-01 (March 1989).

[2] M. Basile, G Cara Romeo, L. Cifarelli, A. Contin, G. D'Ali, P. Di Cesare, B. Esposito, P. Giusti, T. Massam, F. Palmonari, G. Sartorelli, G. Valenti and A. Zichichi, Phys. Lett. 92B (1980) 367.

[3] M. Basile et al., Nuovo Cimento 58A (1980) 193.

[4] M. Basile et al., Phys. Lett. 95B (1980) 311.

[5] M. Basile et al., Lettere al Nuovo Cimento 29 (1980) 491.

[6] M. Basile et al., Phys. Lett. 99B (1981) 247.

[7] M. Basile et al., Lettere al Nuovo Cimento 30 (1981) 389.

[8] M. Basile et al., Lettere al Nuovo Cimento 31 (1981) 273.

[9] M. Basile et al., Nuovo Cimento 65A (1981) 400.

[10] M. Basile et al., Nuovo Cimento 65A (1981) 414.

[11] M. Basile et al., Lettere al Nuovo Cimento 32 (1981) 210.

[12] M. Basile et al., Lettere al Nuovo Cimento 32 (1981) 321.

[13] M. Basile et al., Nuovo Cimento 66A (1981) 129.

[14] M. Basile et al., Nuovo Cimento 67A (1982) 53.

[15] M. Basile et al., Nuovo Cimento 67A (1982) 244.

[16] M. Basile et al., Nuovo Cimento 73A (1983) 329.

[17] M. Basile et al., Lettere al Nuovo Cimento 36 (1983) 303.

[18] M. Basile et al., Lettere al Nuovo Cimento 36 (1983) 555.

[19] M. Basile et al., Lettere al Nuovo Cimento 36 (1983) 563.

[20] M. Basile et al., Lettere al Nuovo Cimento 37 (1983) 246.

[21] M. Basile et al., Lettere al Nuovo Cimento 37 (1983) 289.

[22] M. Basile et al., Lettere al Nuovo Cimento 38 (1983) 289.

[23] M. Basile et al., Lettere al Nuovo Cimento 38 (1983) 359.

[24] M. Basile et al., Lettere al Nuovo Cimento 38 (1983) 367.

[25] M. Basile et al., Nuovo Cimento 79A (1984) 1.

[26] M. Basile et al., Lettere al Nuovo Cimento 41 (1984) 293.

[27] M. Basile et al., Lettere al Nuovo Cimento 41 (1984) 298.

[28] G. Ingelman, Proc. of the Workshop on "Physics at HERA", DESY, Hamburg, Germany, 29-30 October 1991 (Ed. W. Buchmüller and G. Ingelman), v.3, p. 1366, and preprint TSL ISV 92-0065 (1992).

[29] G. Marchesini and B. R. Webber, Nucl. Phys B130 (1988) 571.

[30] T. Sjöstrand, Comp. Phys. Comm. 39 (1986) 347; T. Sjöstrand and M. Bengtsson, Comp. Phys. Comm. 43 (1987) 367; H. U. Bengtsson and T. Sjöstrand, Comp. Phys. Comm. 46 (1987) 43; T. Sjöstrand and M. van Zijl, Phys. Rev. D36 (1987) 2019; T. Sjöstrand, preprint CERN-TH.6488/92, May 1992.

[31] F. Anselmo, L. Cifarelli, E. Eskut and Yu. M. Shabelski, Nuovo Cimento 105A (1992) 1371.

[32] EMC Collaboration (M.Arneodo et al.), Z. Phys. C35 (1987) 433.

[33] E. Eichten, I. Hinchliffe, K. Lane and C. Quigg, Rev. Mod. Phys. 56 (1984) 579; 58 (1985) 1065.

[34] J. G. Morfin and W.-K. Tung, Z. Phys. C.52 (1991) 13. 
[35] L. Cifarelli, E. Eskut and Yu. M. Shabelski, Nuovo Cimento 106A (1993) 389.

[36] L. Cifarelli, Yu. M. Shabelski and O. P. Strogova: "Monte Carlo simulations for leading proton production in $p p$ interactions up to $\sqrt{s}=200 \mathrm{TeV}$ ", preprint EMC'SC! in preparation. 


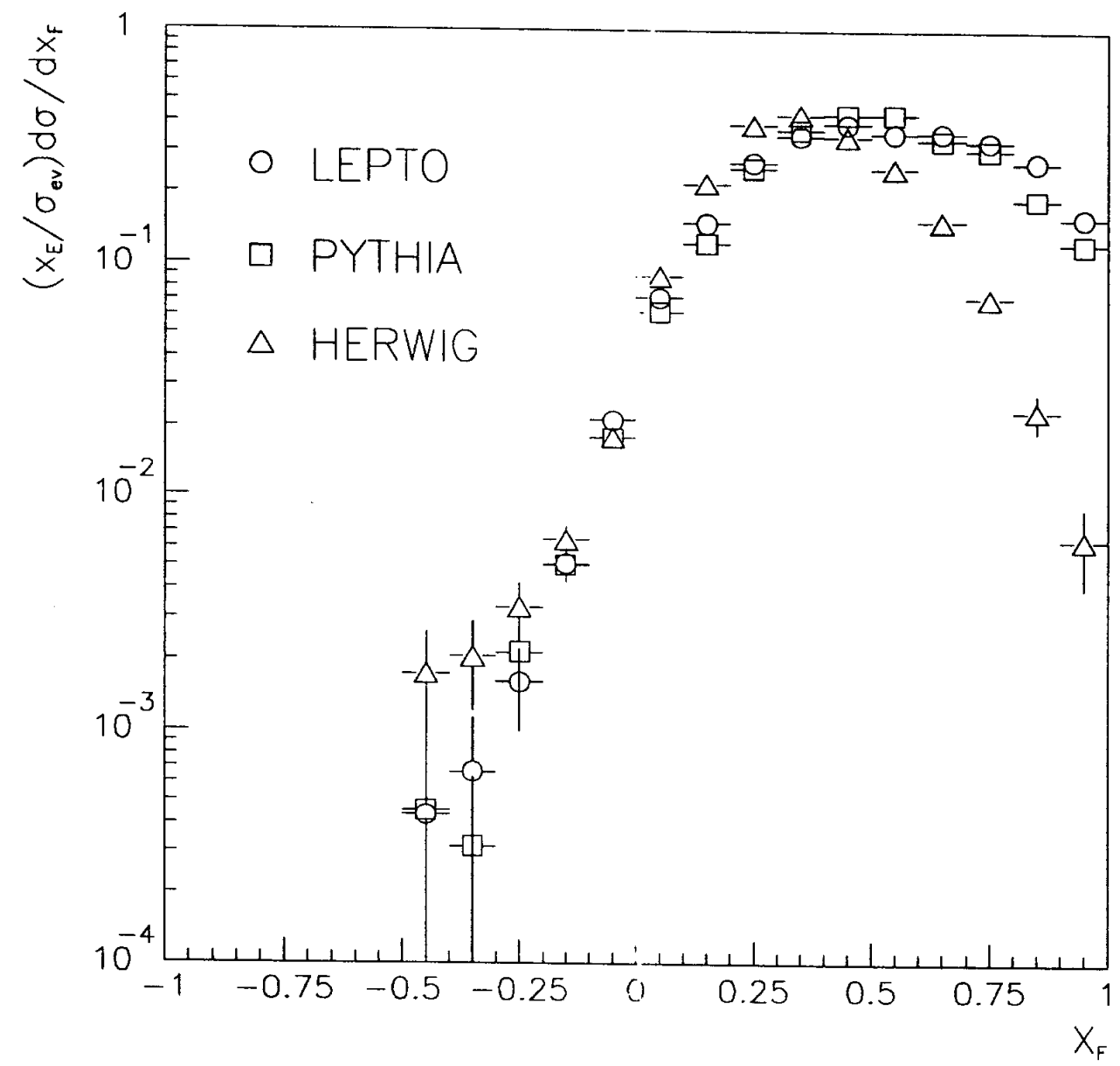

Fig. 1a 


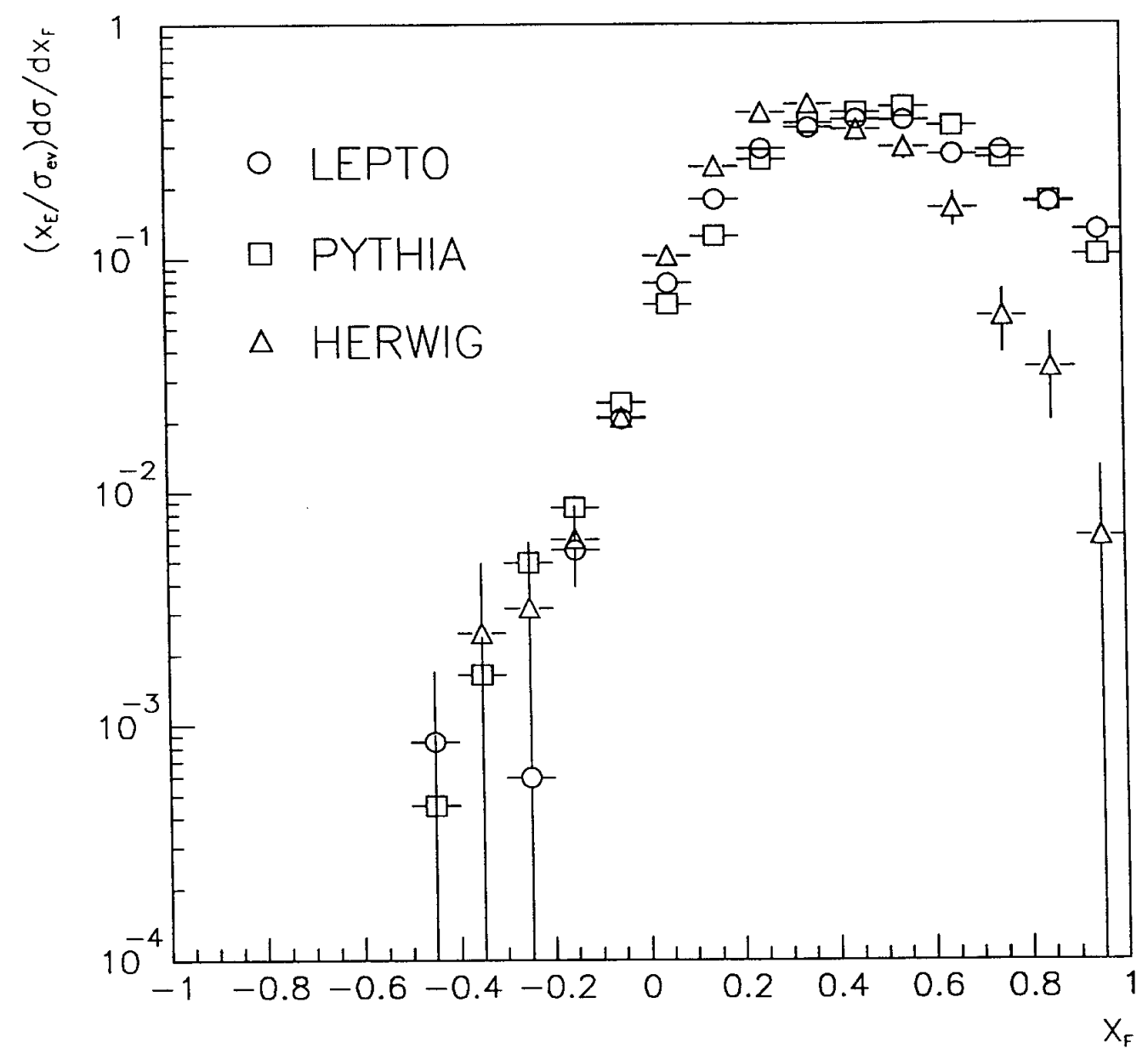

Fig. $1 \mathrm{~b}$ 


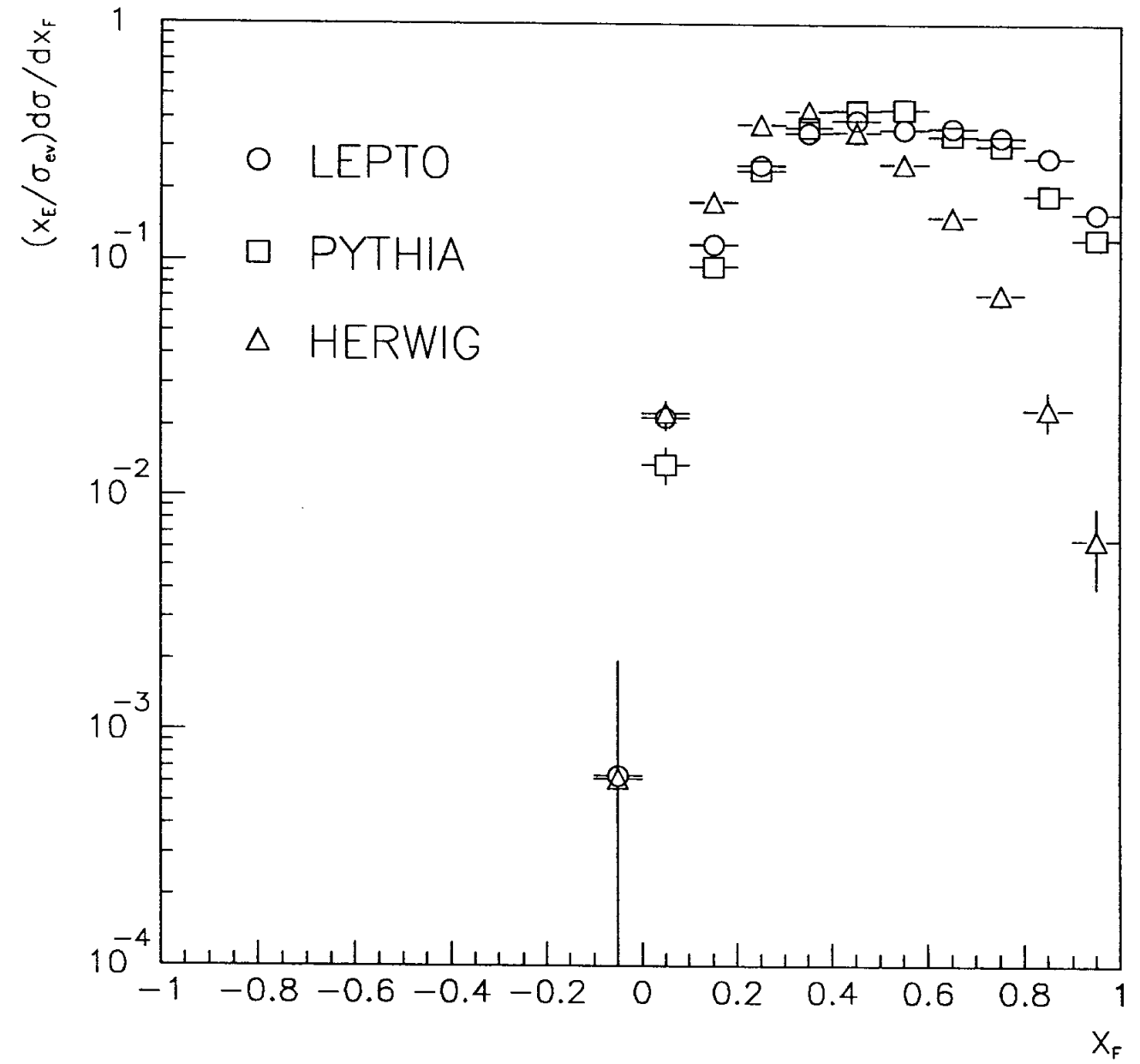

Fig. 1c 


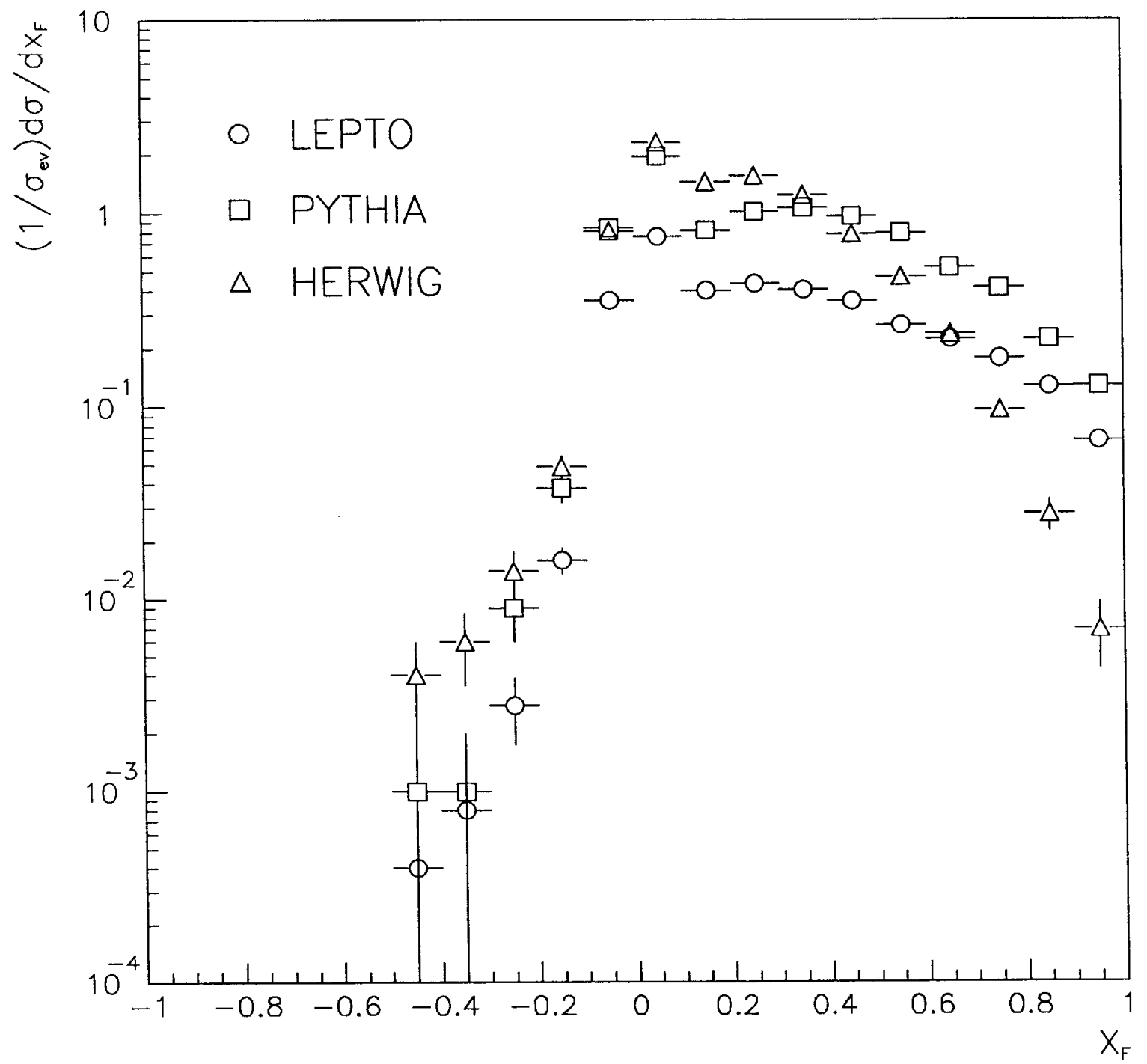

Fig.1d 


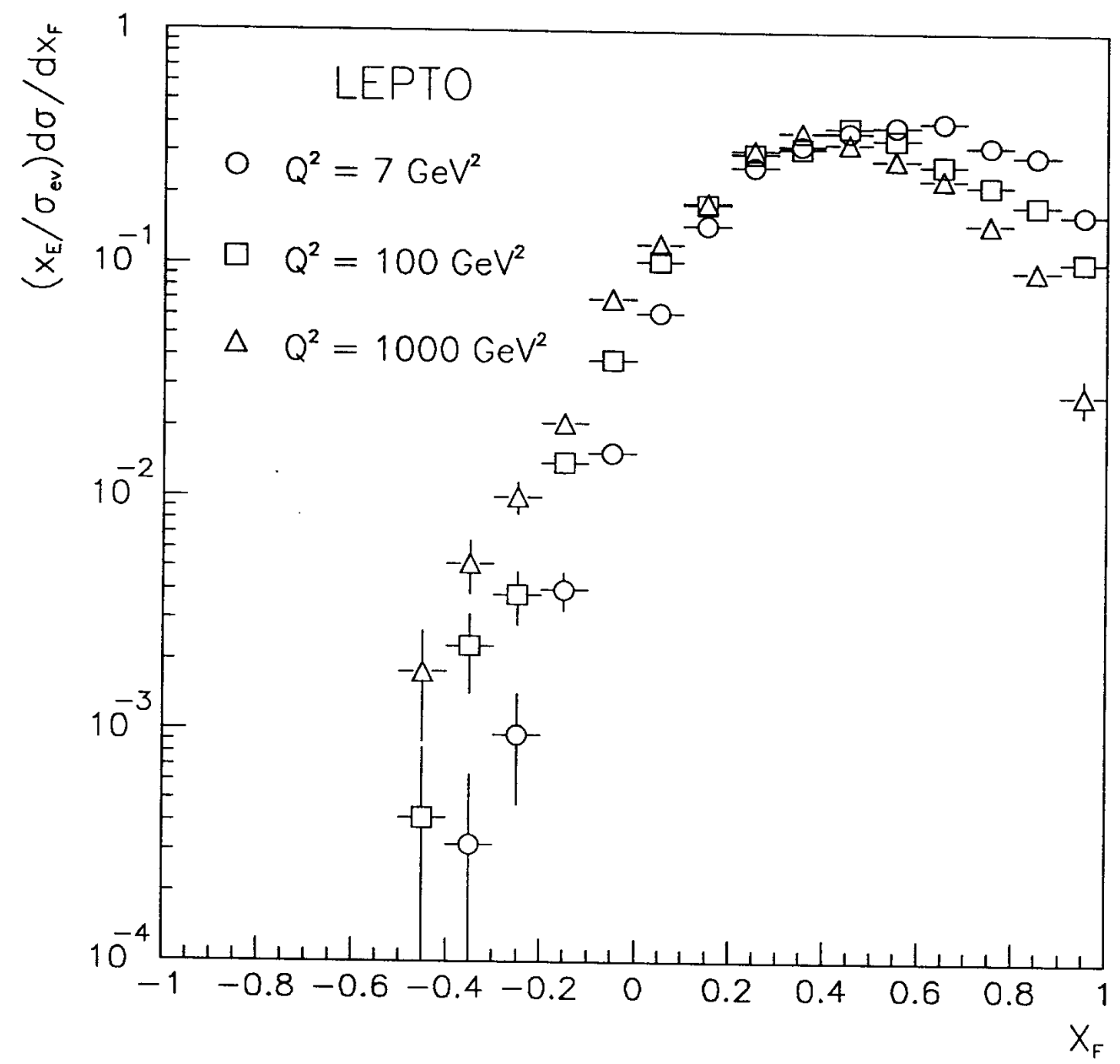

Fig.2a 


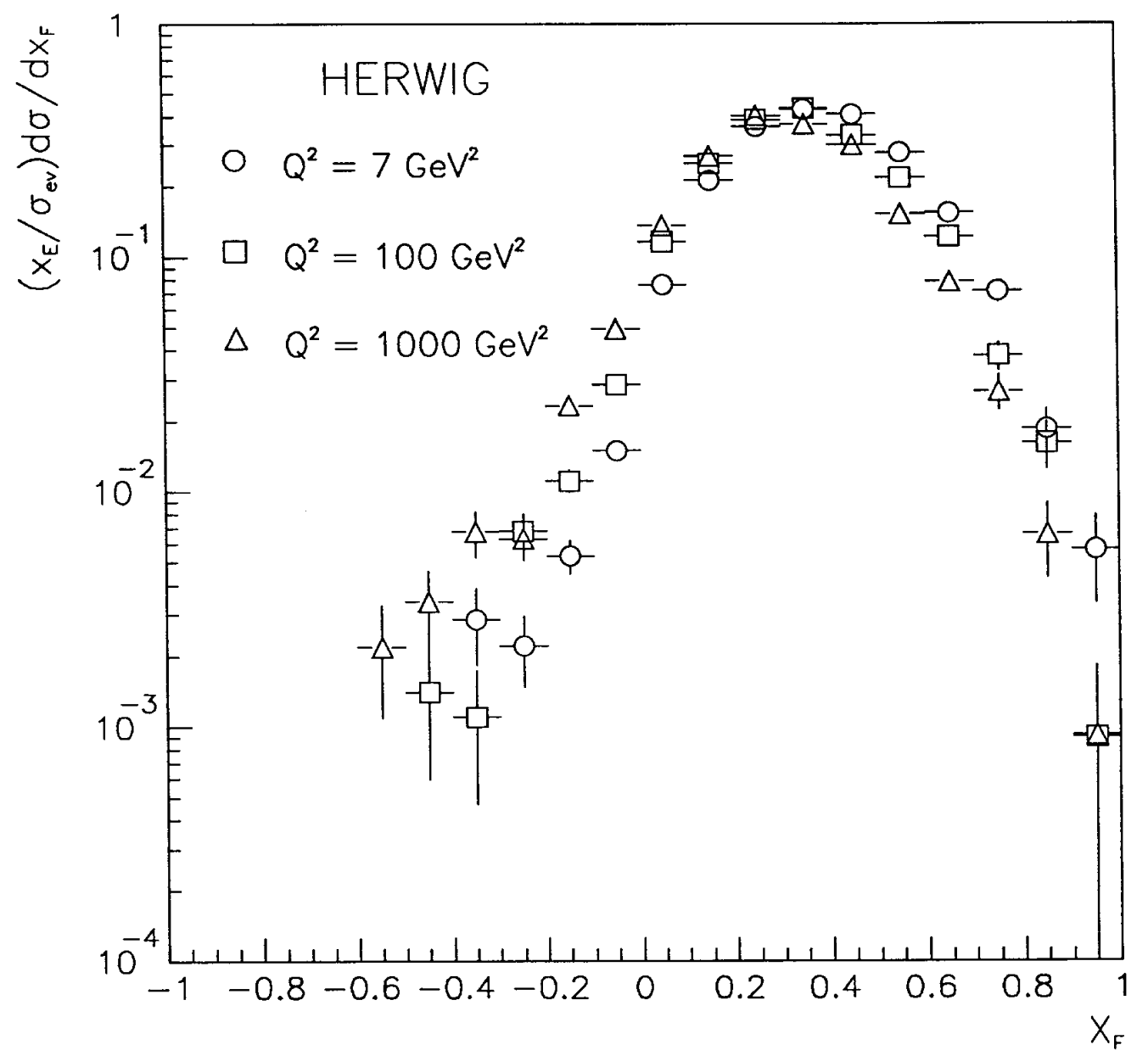

Fig. $2 b$ 


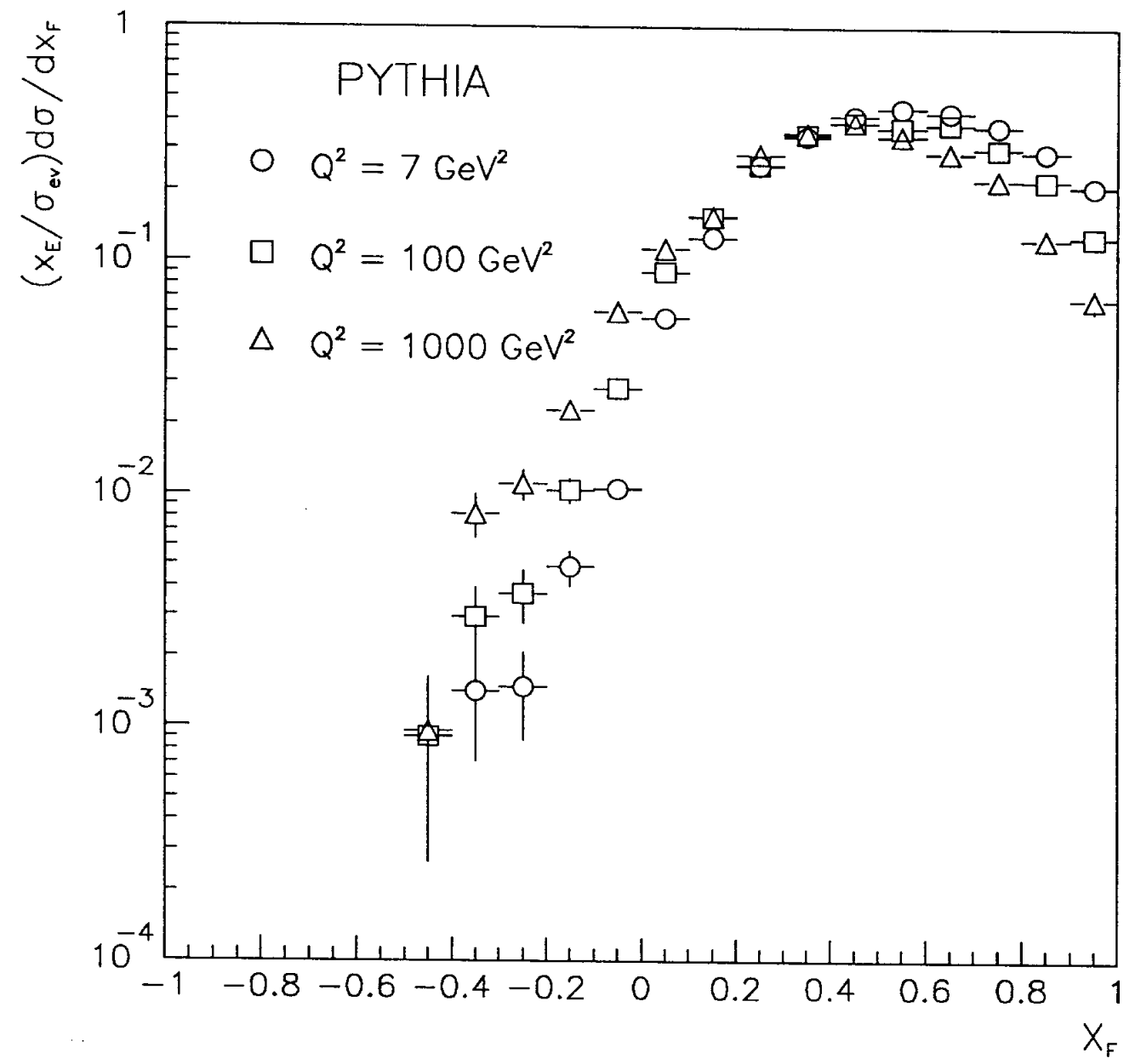

Fig.2c 


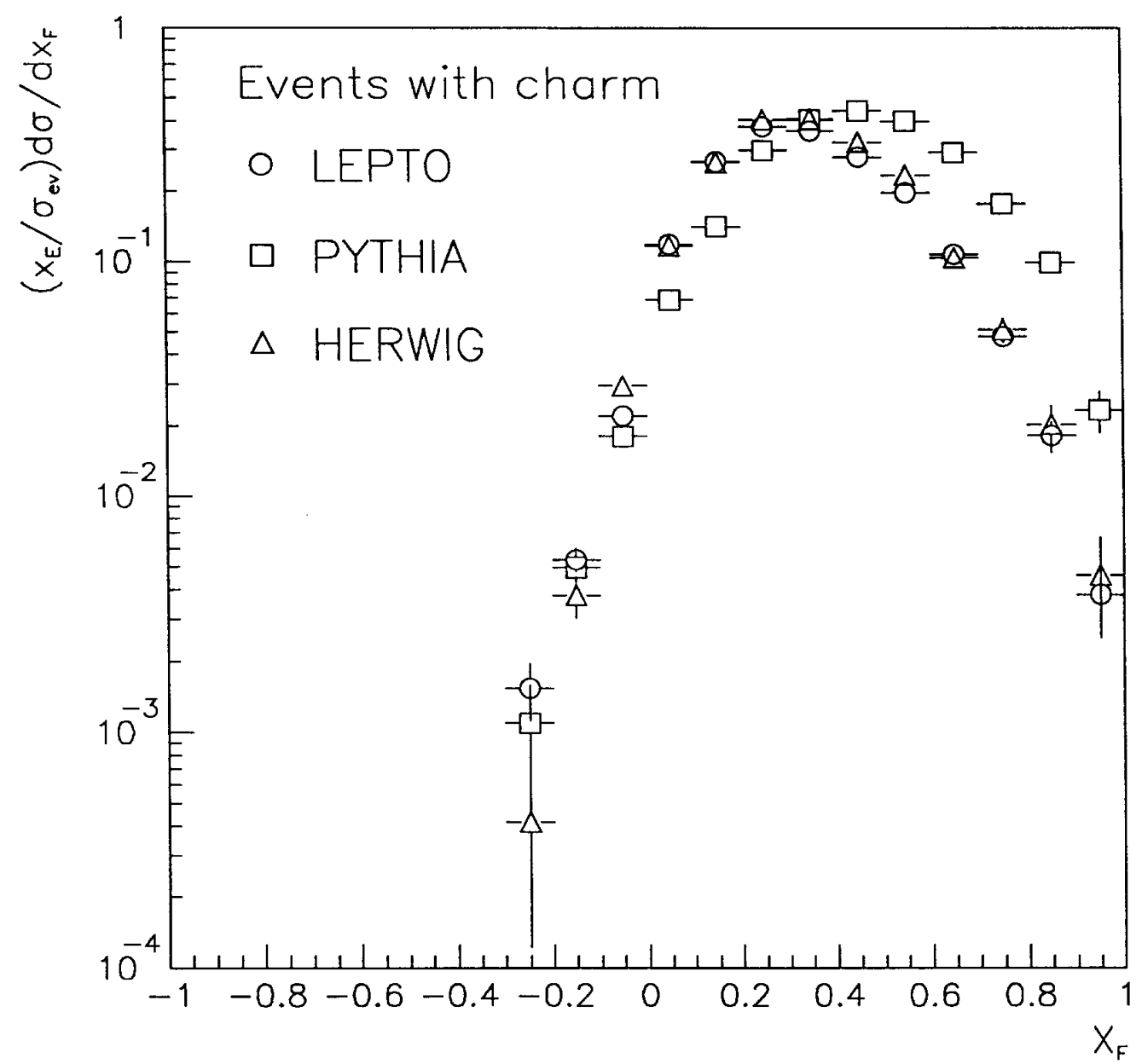

Fig.3a 


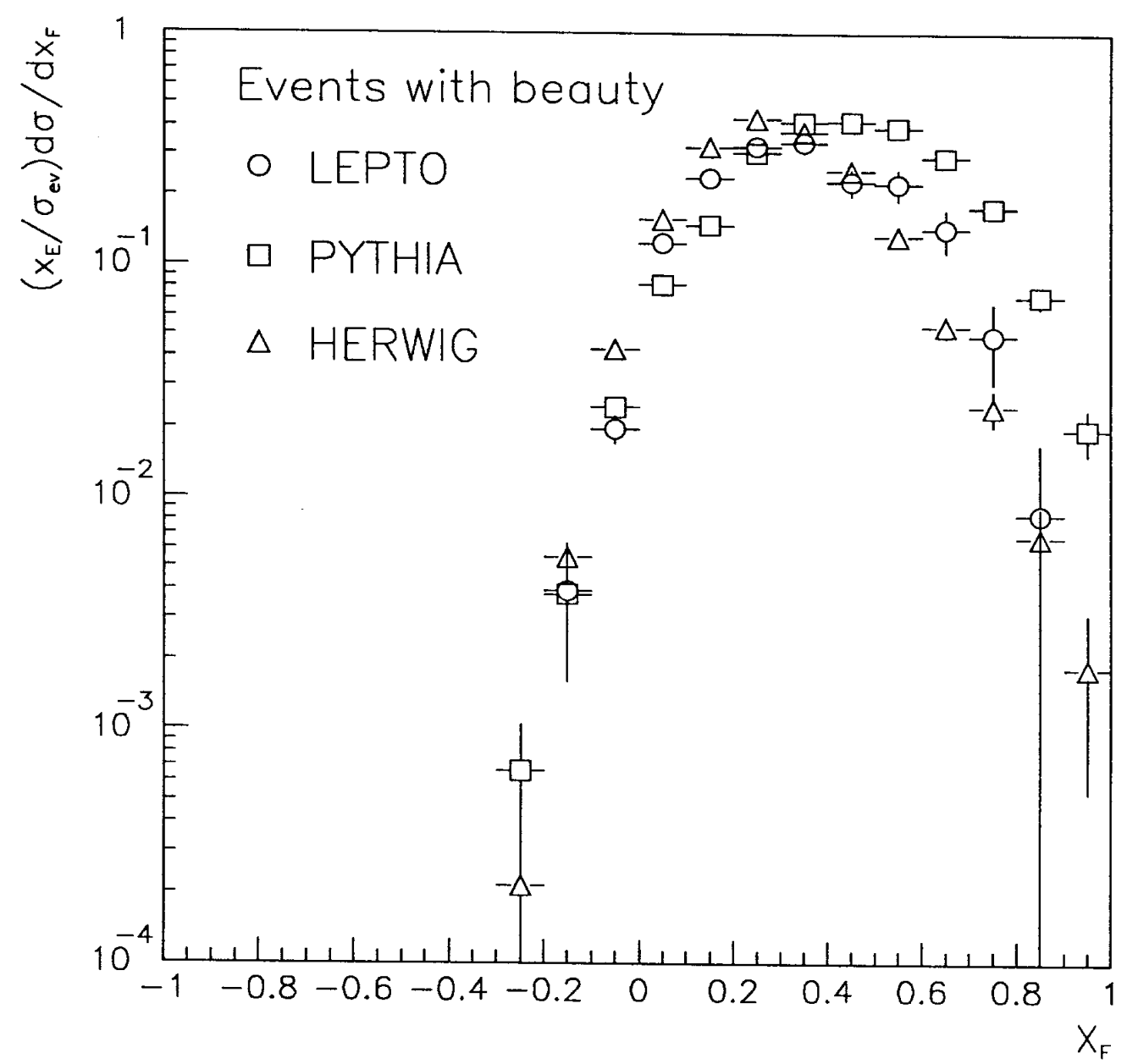

Fig. 3b 


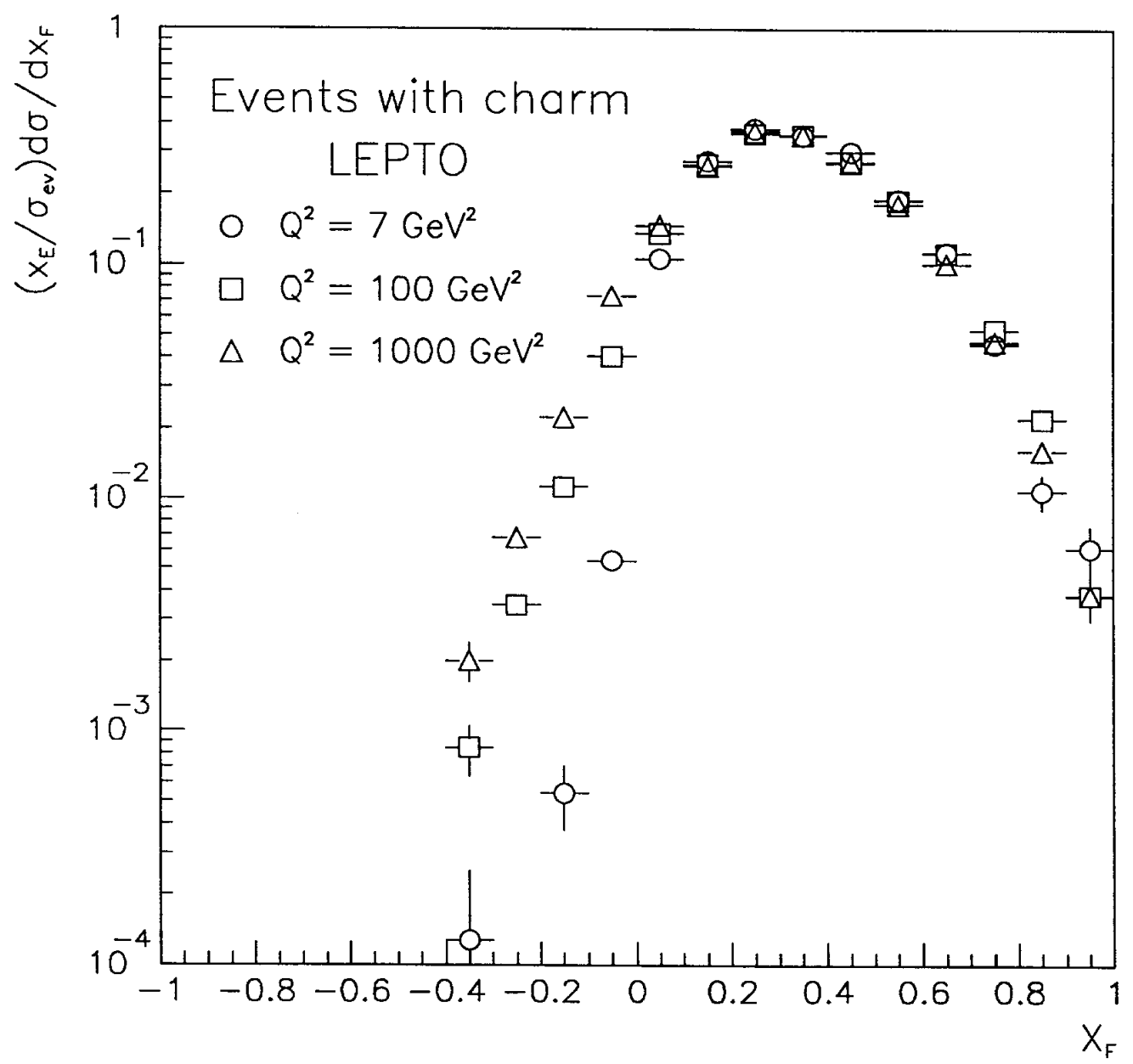

Fig.4 


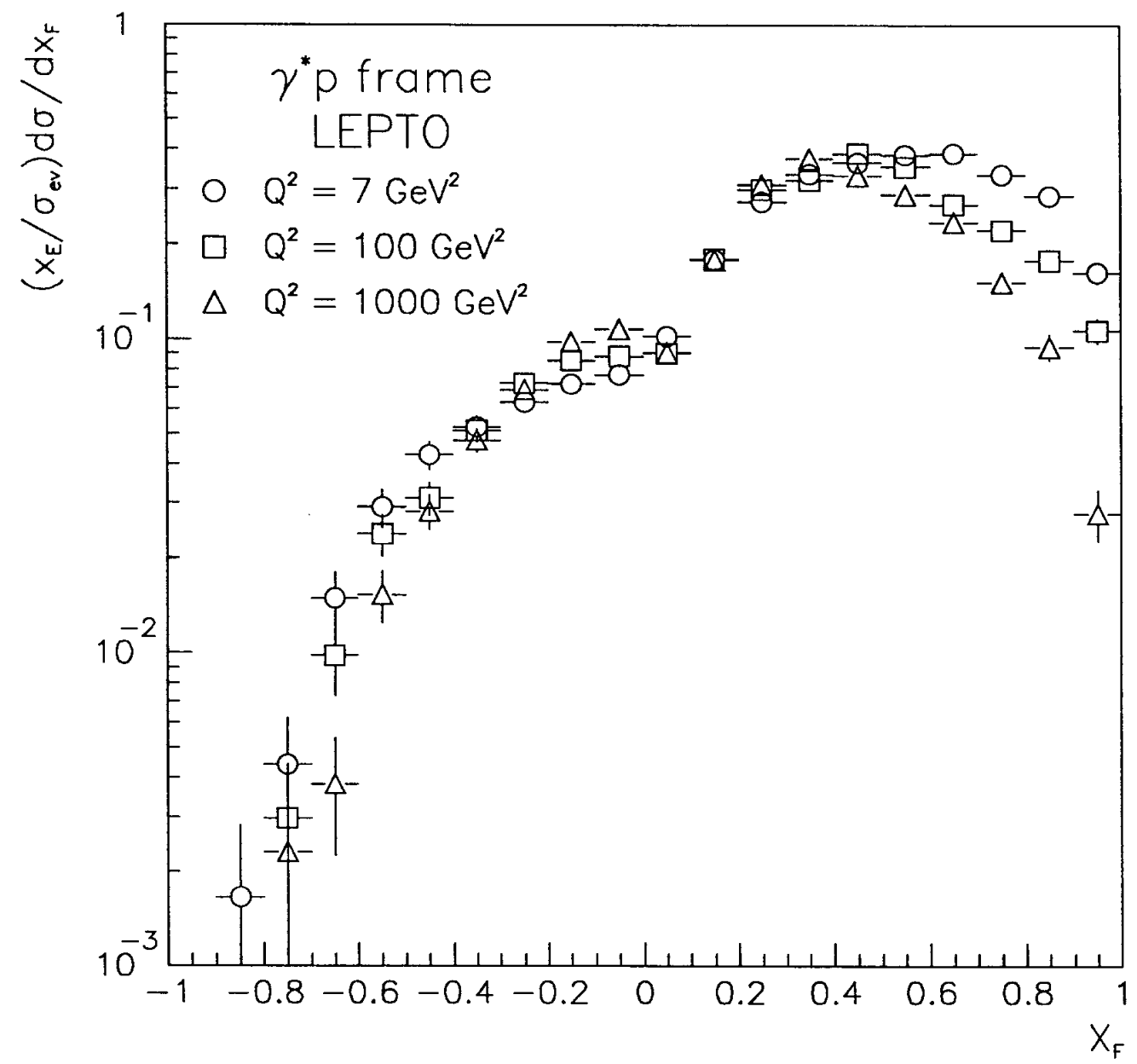

Fig.5a 


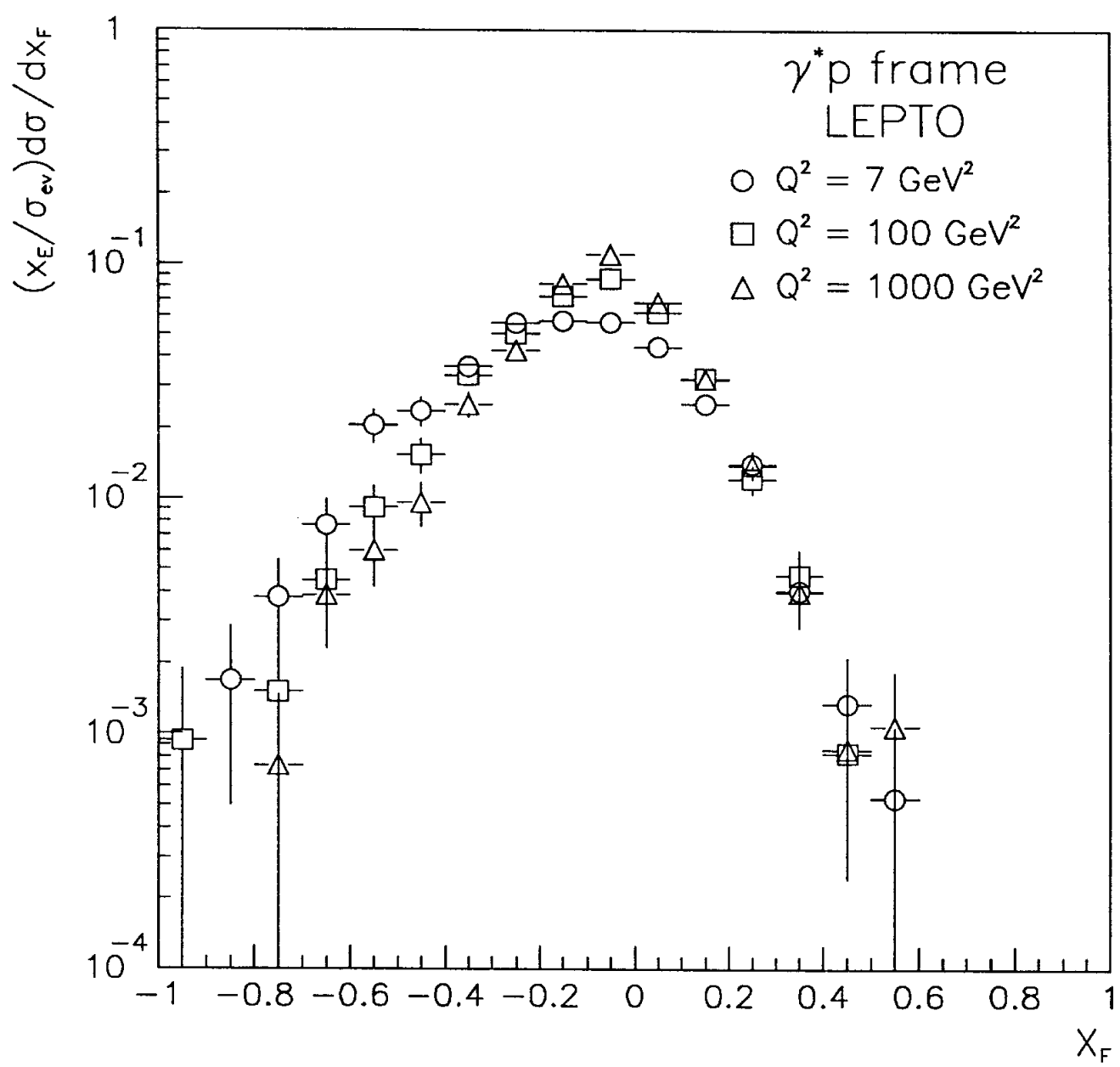

Fig. 5b 


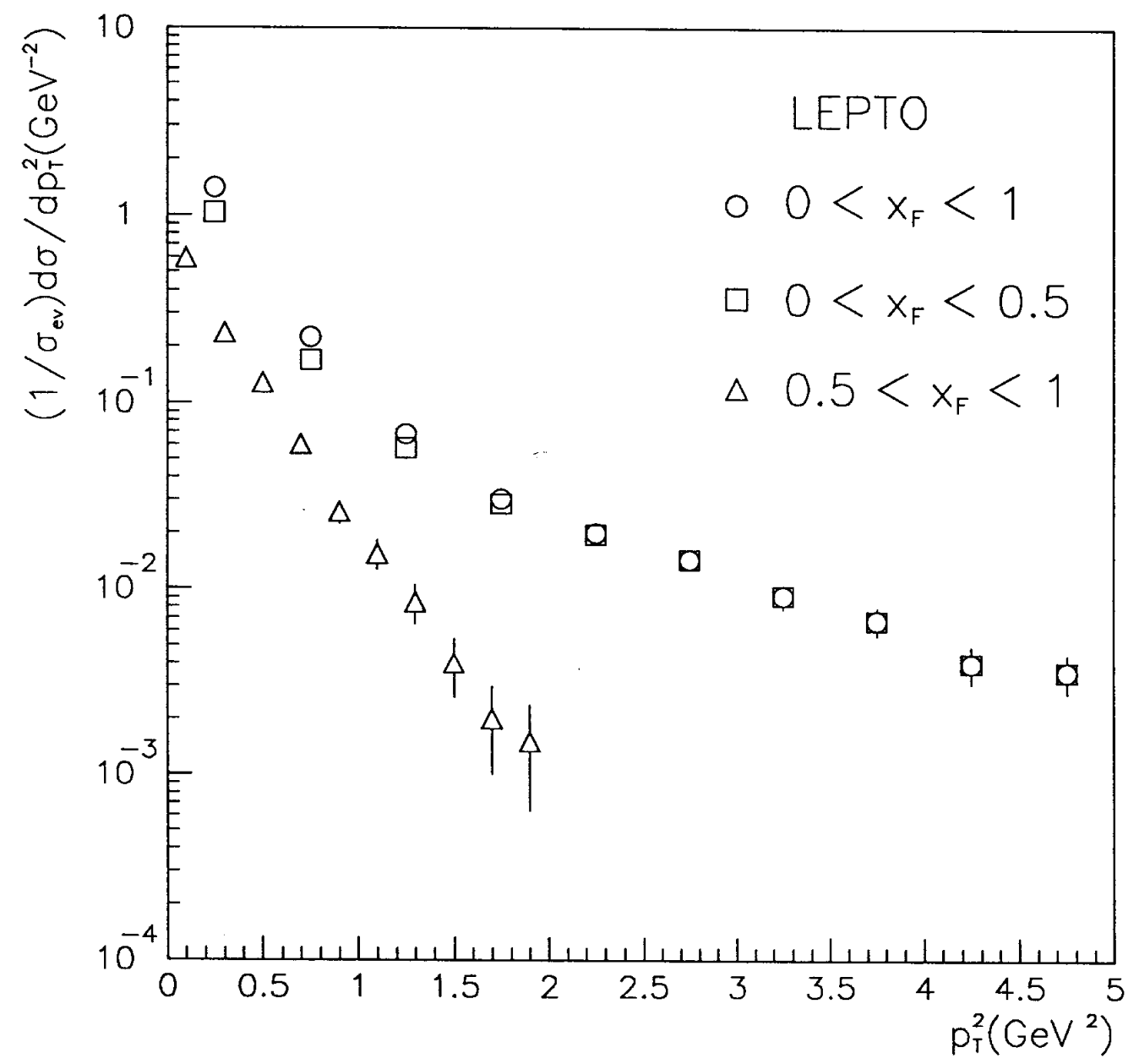

Fig. 6a 


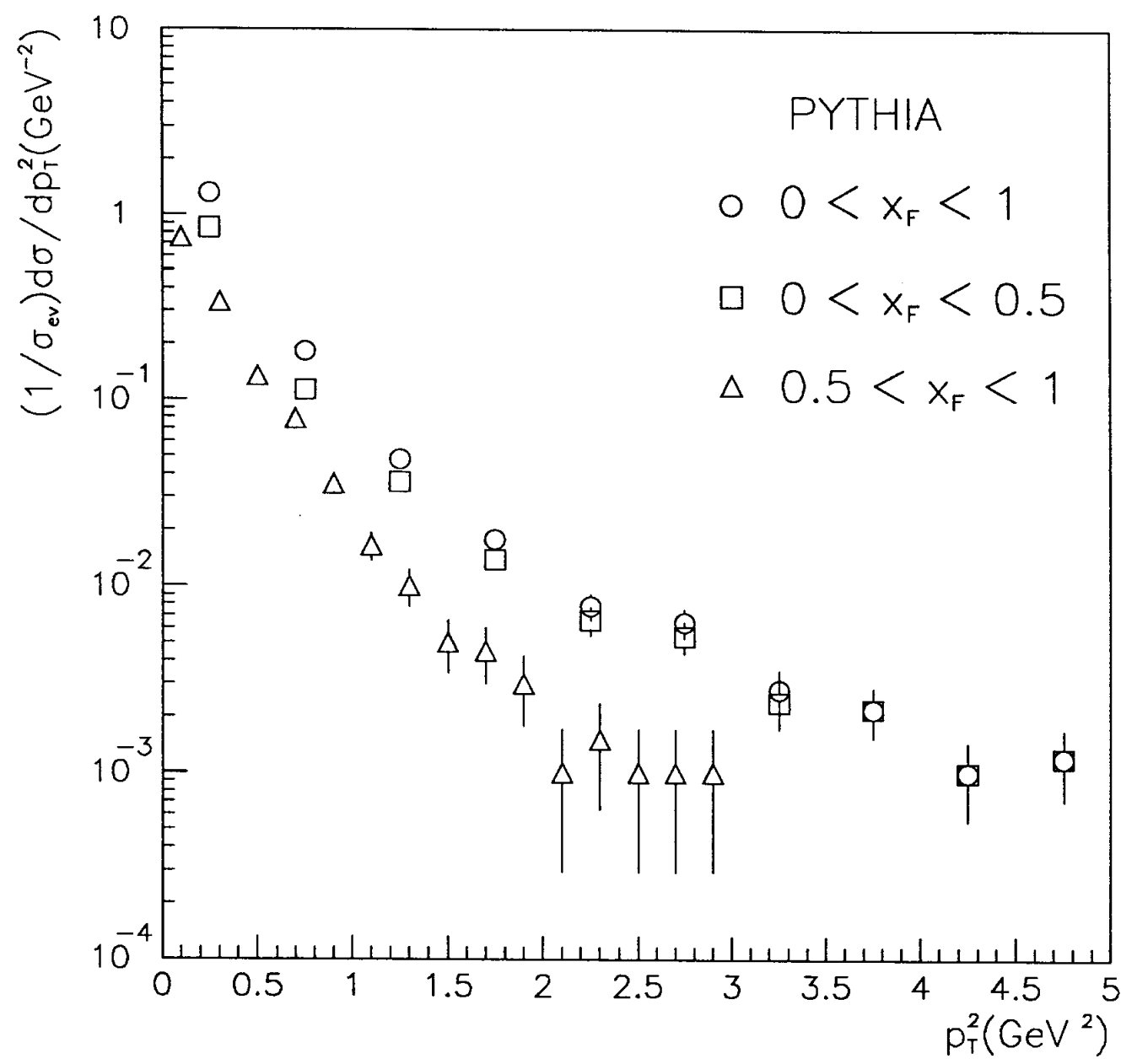

Fig. 6b 


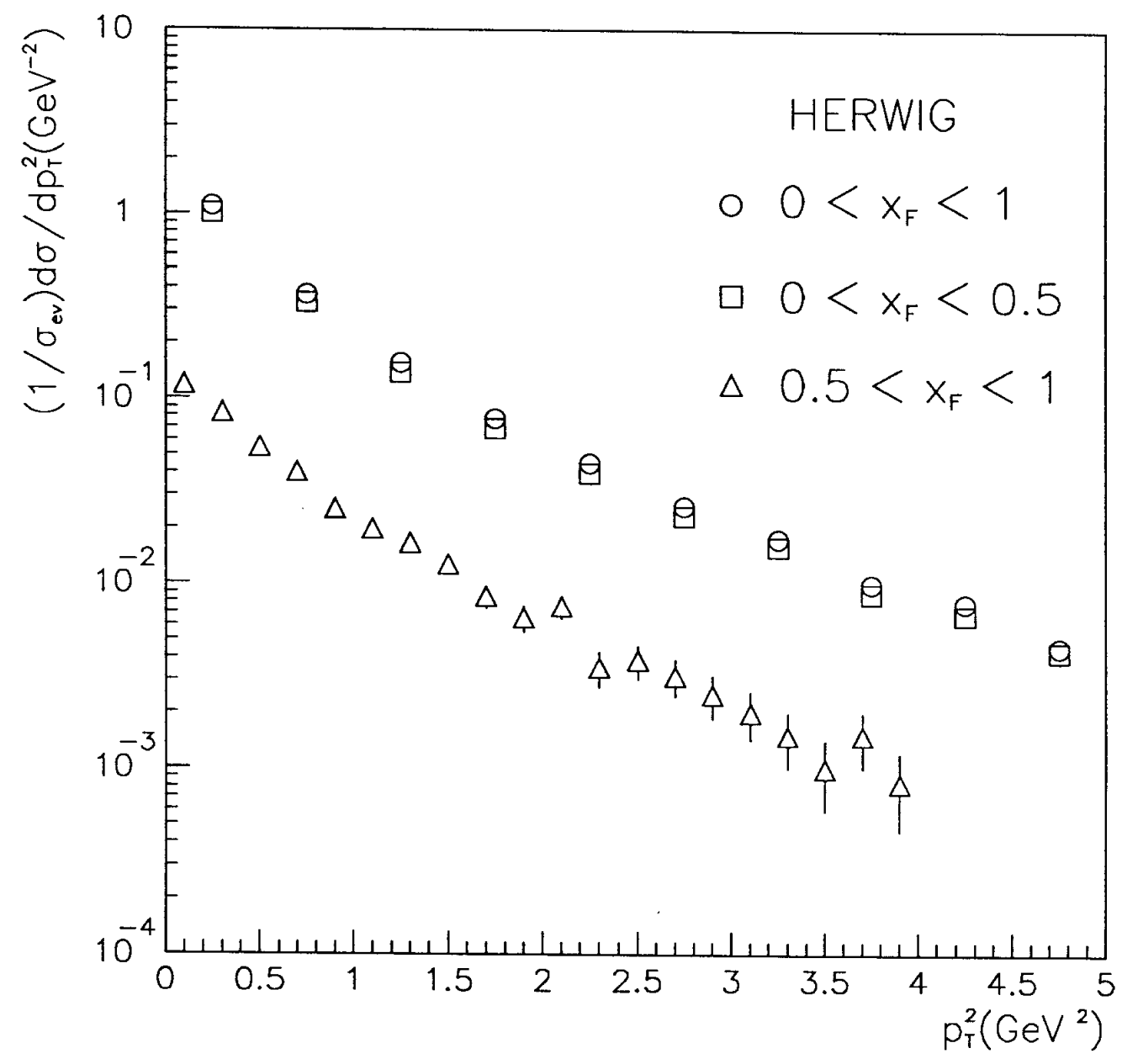

Fig.6c 


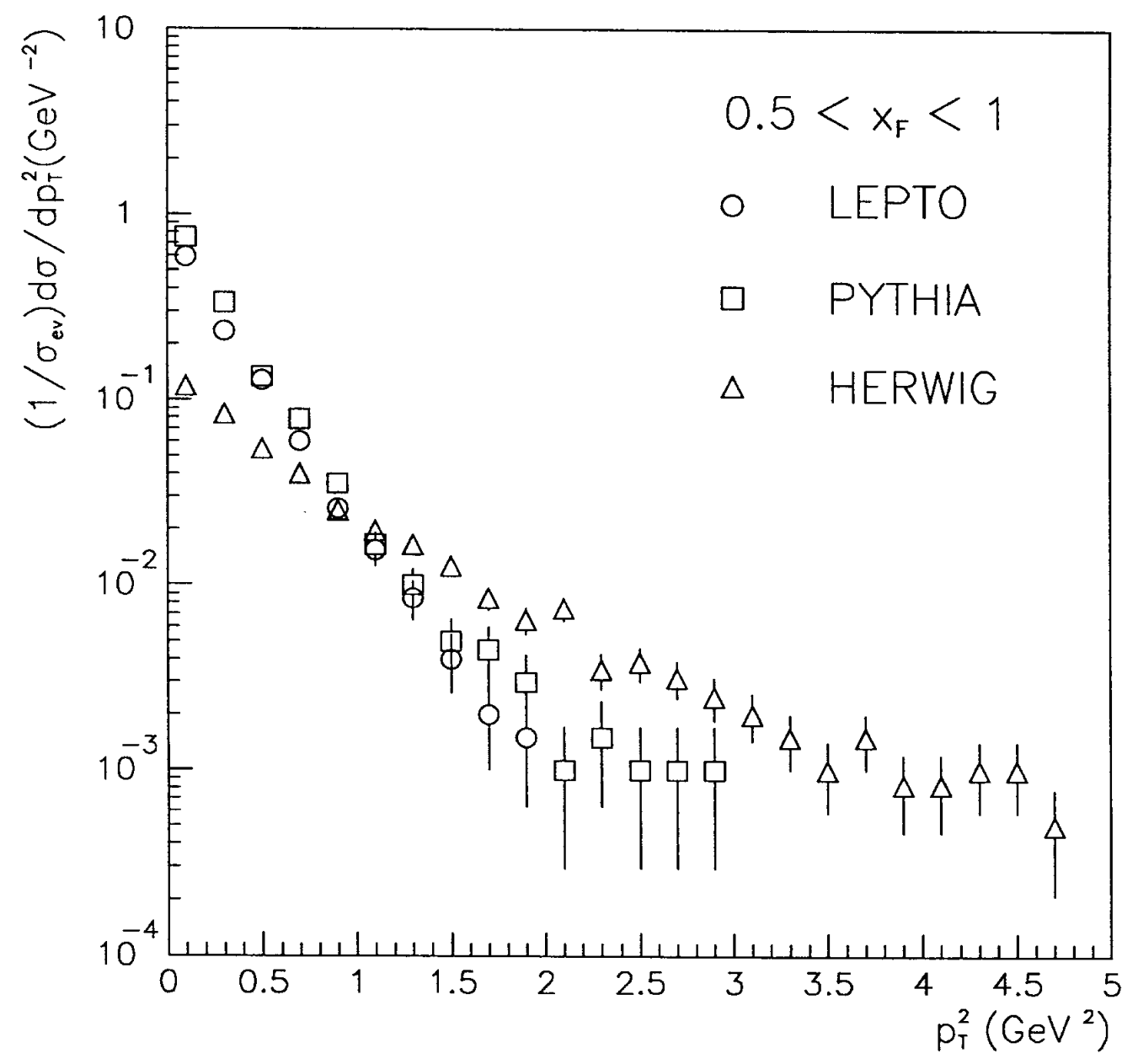

Fig. 6d 


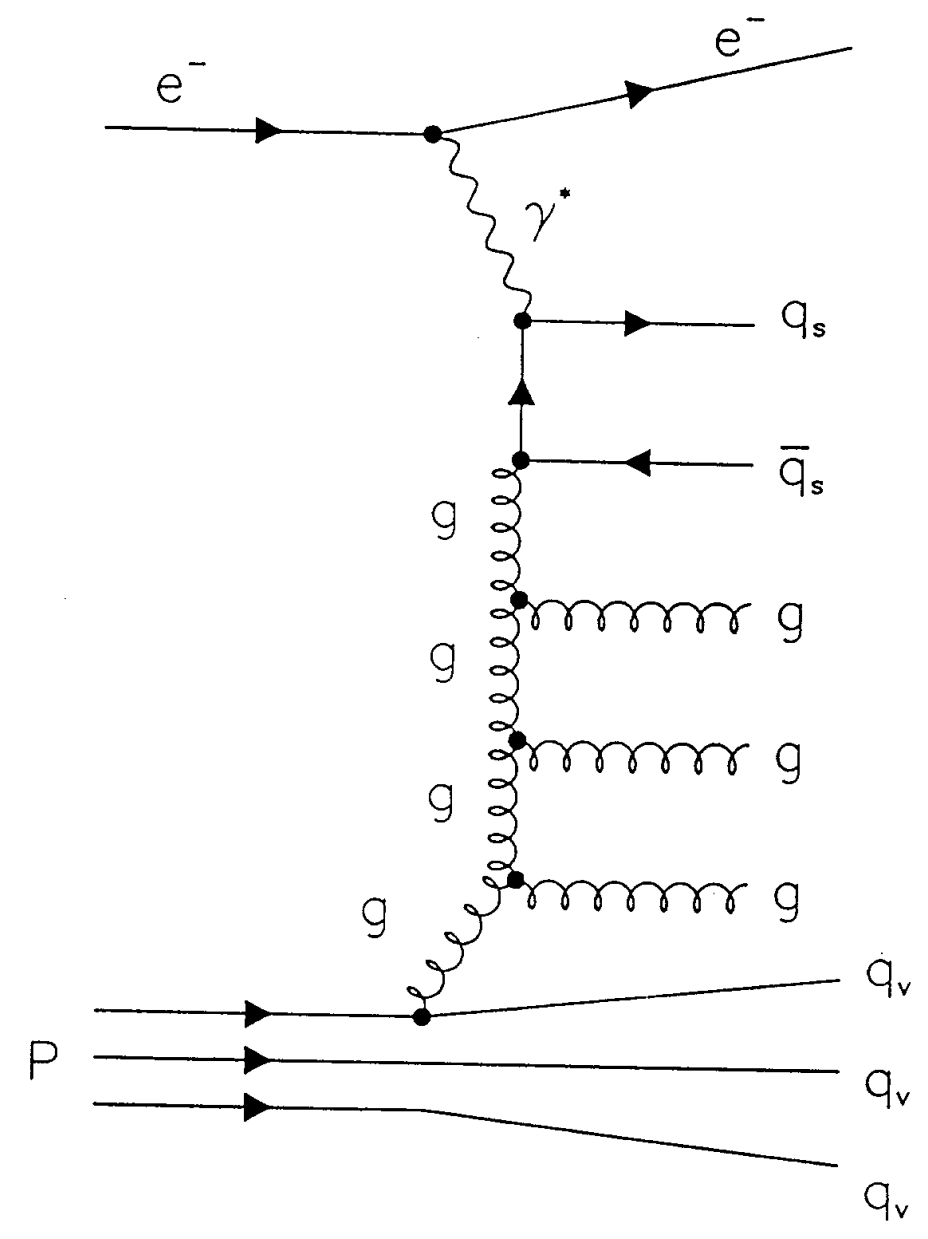

Fig.7 


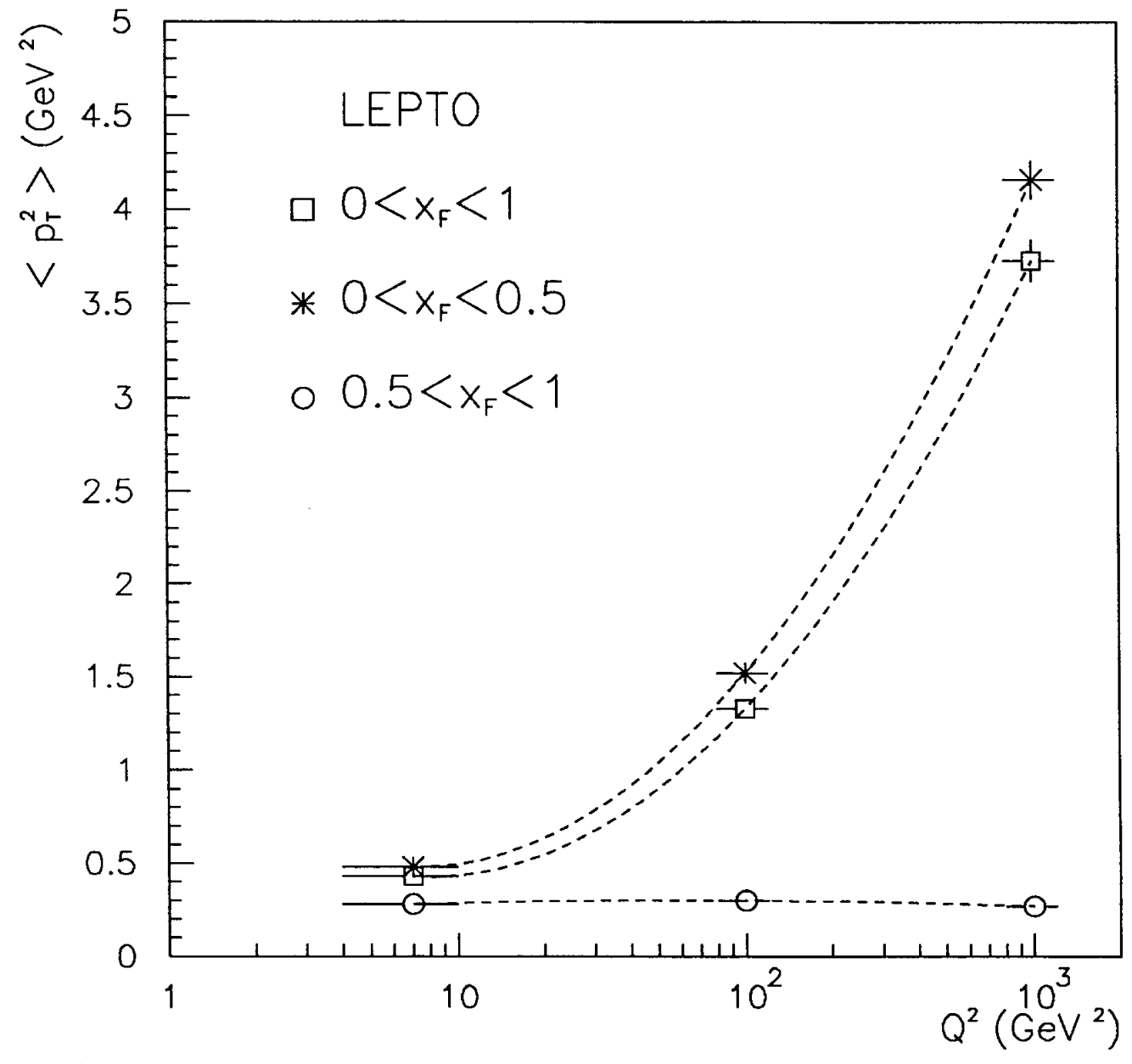

Fig. 8a 


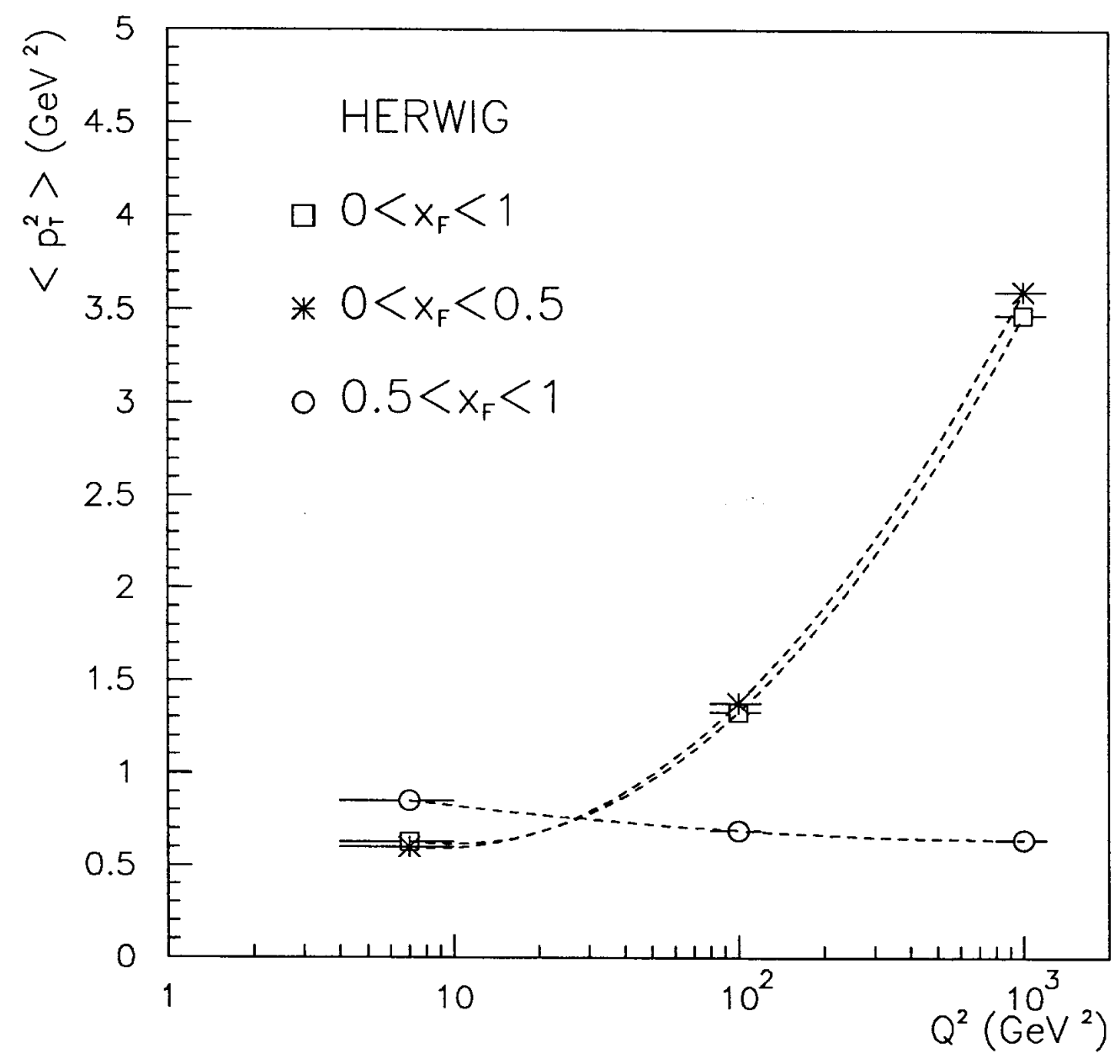

Fig. 8b 


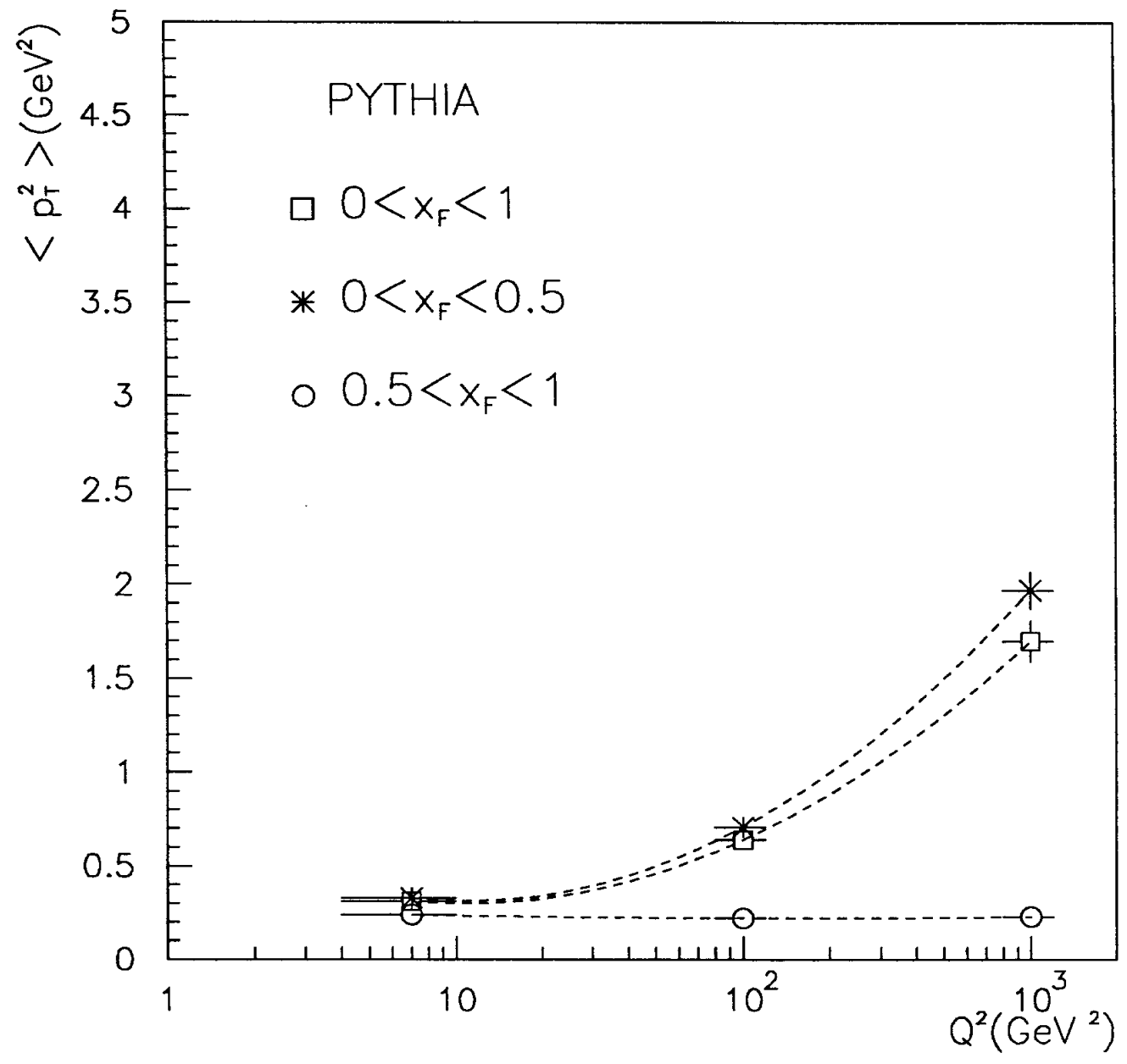

Fig. 8c 


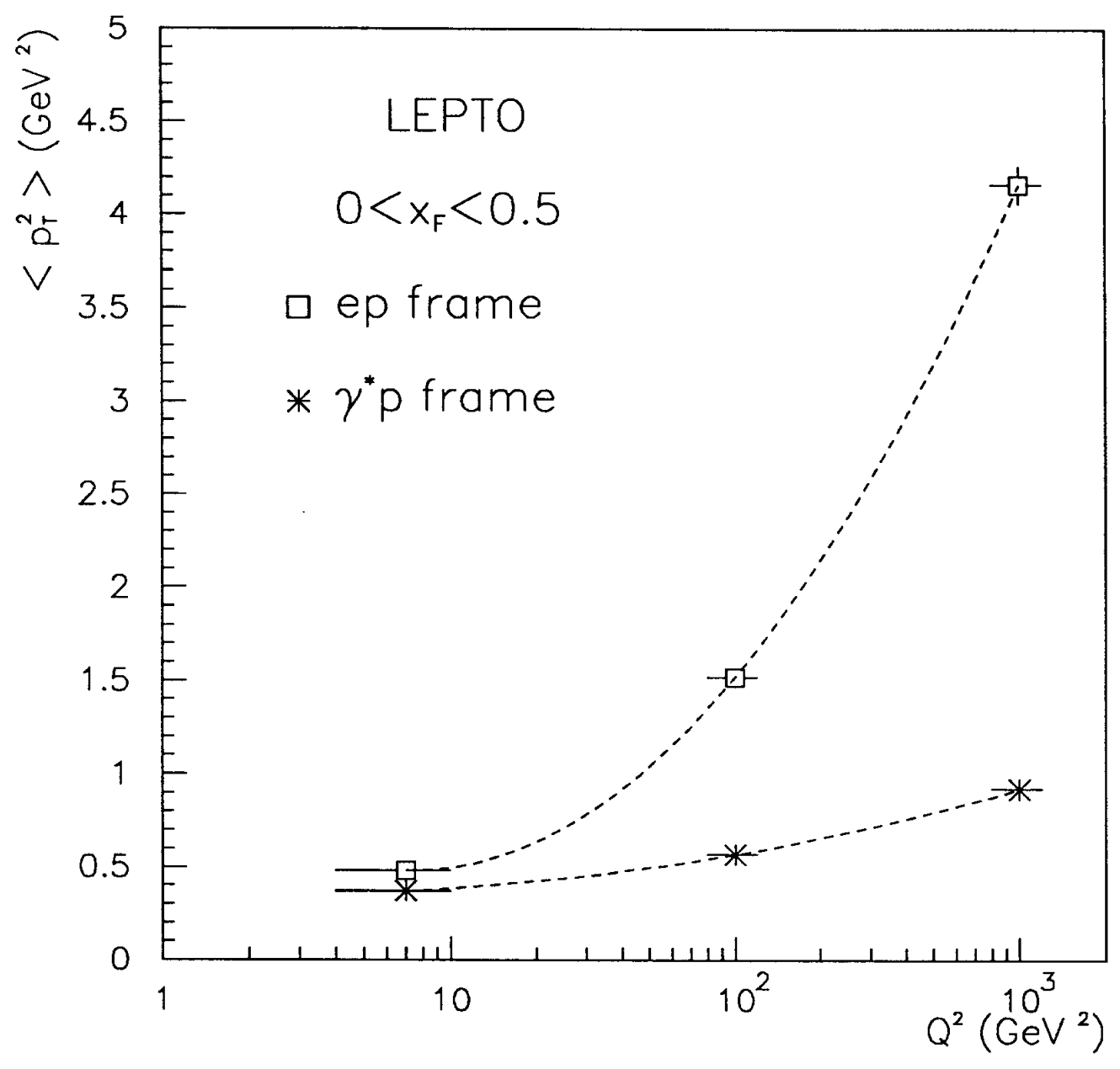

Fig. 9a 


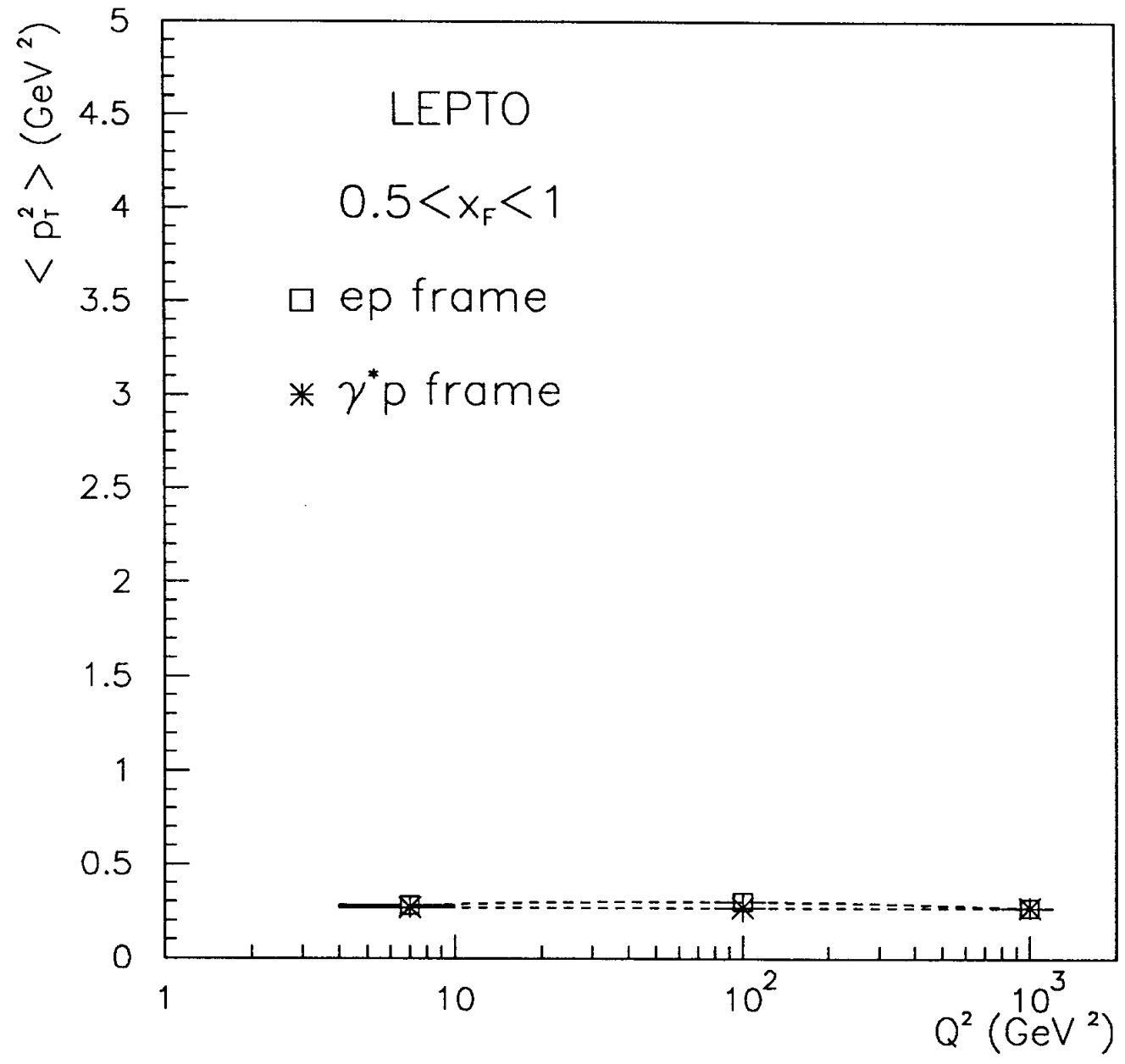

Fig. 9b 


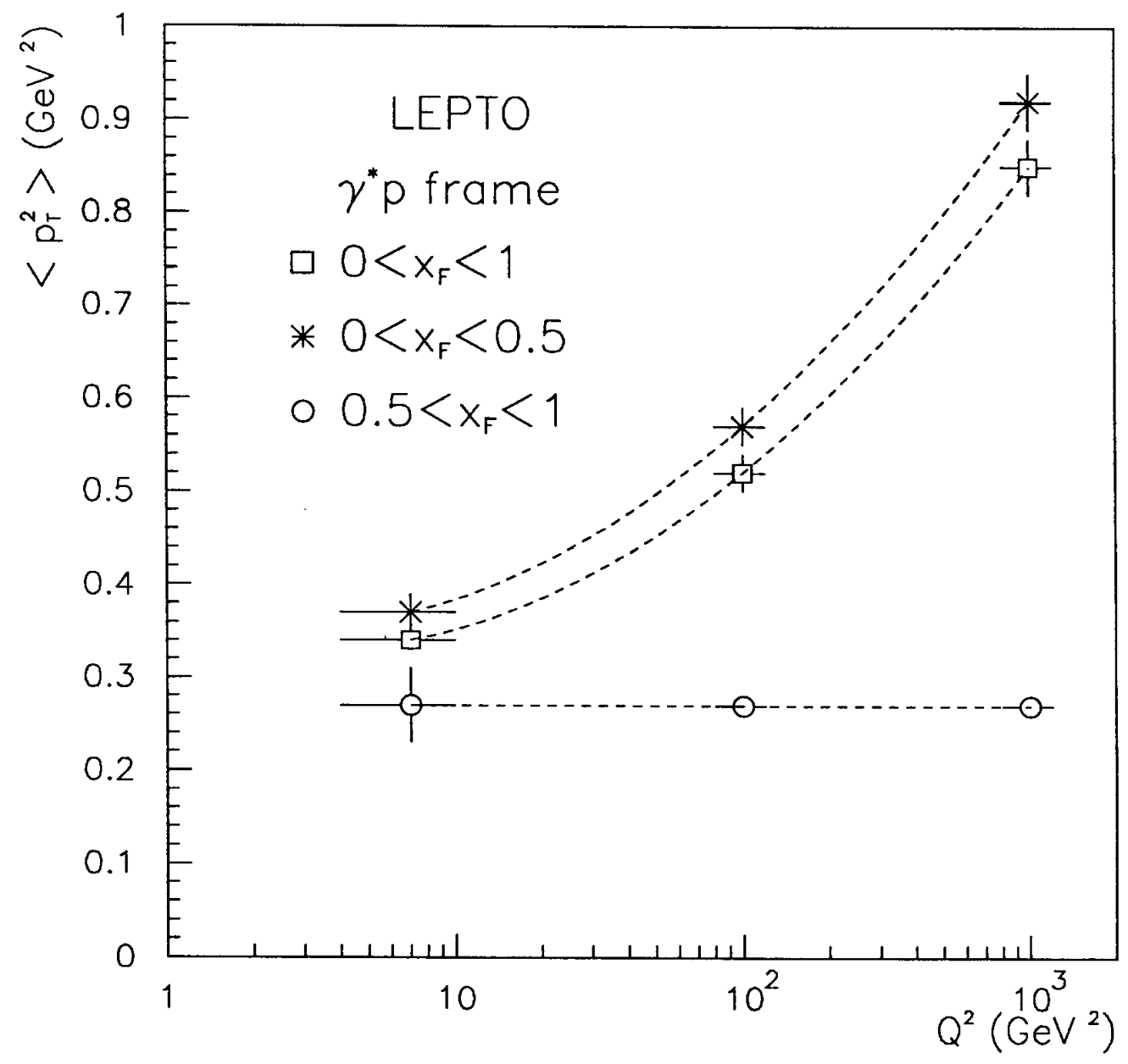

Fig. 10 


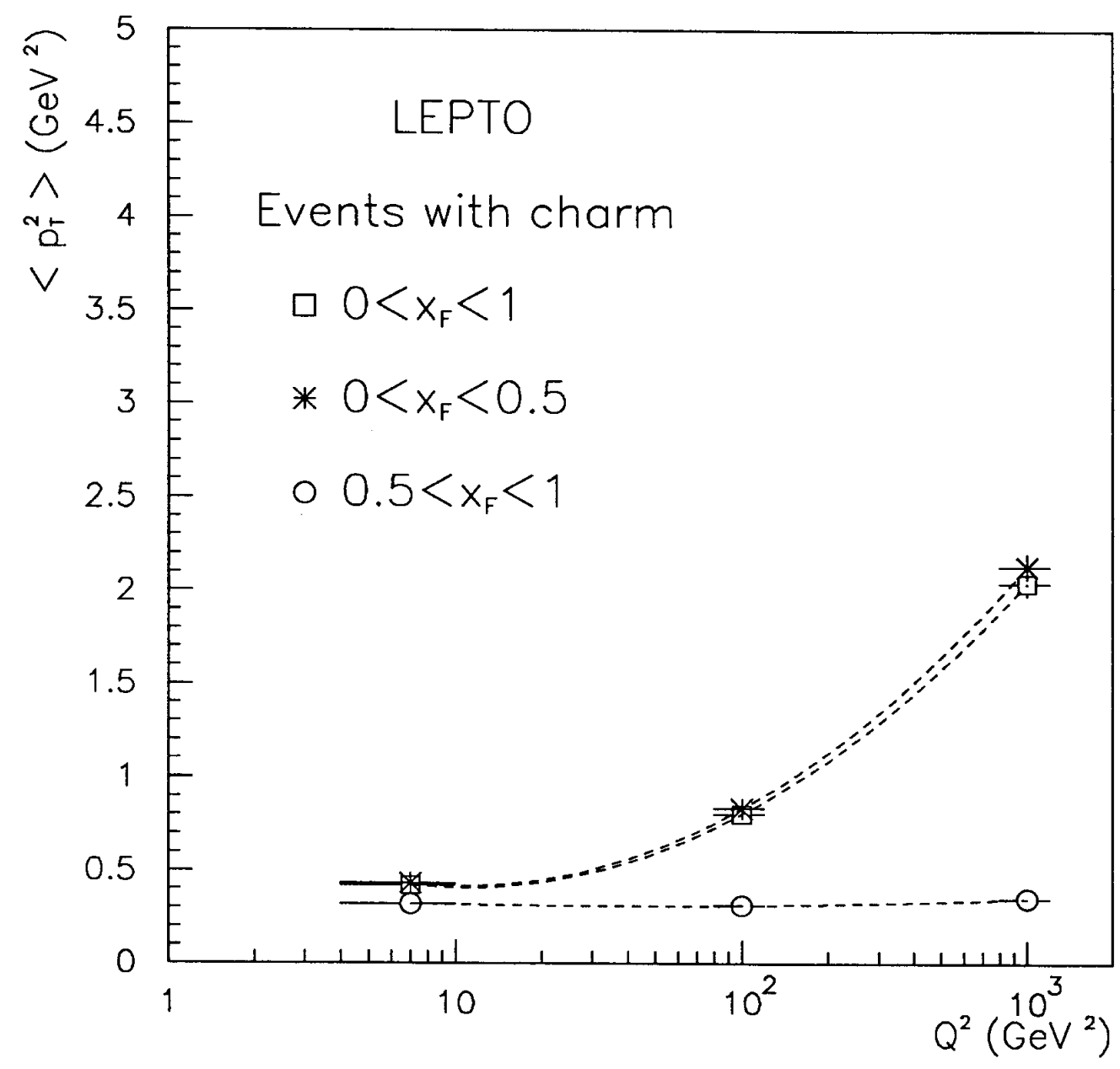

Fig. 11a 


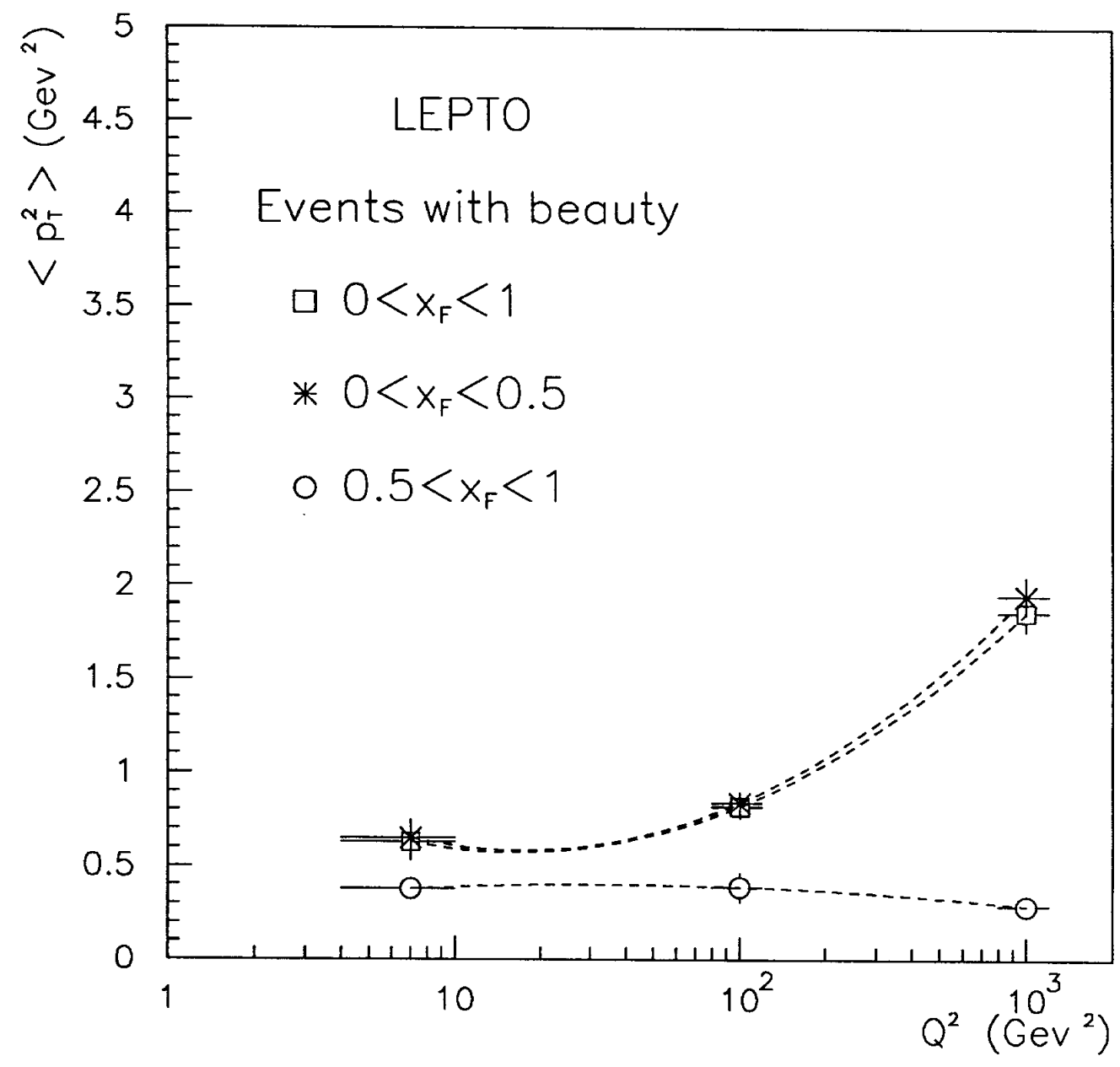

Fig. $11 b$ 